\title{
Endophytism of Penicillium Species in Woody Plants
}

\author{
Rosario Nicoletti ${ }^{1, *}$, Antonio Fiorentino ${ }^{2}$ and Monica Scognamiglio ${ }^{2}$ \\ ${ }^{1}$ Consiglio per la Ricerca e la Sperimentazione in Agricoltura, Unità di Ricerca CAT, Scafati 84018, Italy \\ ${ }^{2}$ Dipartimento di Scienze e Tecnologie Ambientali, Biologiche e Farmaceutiche, Seconda Università di Napoli, Caserta \\ 81100, Italy
}

\begin{abstract}
The genus Penicillium is ubiquitous and its species are commonly recovered from every kind of substrate and environmental conditions. Therefore, not surprisingly, Penicillium strains are commonly reported in investigations dealing with fungal endophytic assemblages of plants in both natural and anthropic contexts. As such they are implicated in more or less effective relationships with the host, and involved in applicative issues concerning plant protection and the production of bioactive compounds to be exploited as pharmaceuticals. The available data on occurrence, biocenotic role, production and bioactivities of secondary metabolites of Penicillium strains which have been isolated as endophytes from woody plants are reviewed in the present paper in the aim to introduce the state of the art to researchers involved in this particular field.
\end{abstract}

Keywords: Bioactive metabolites, endophytes, fungal diversity, mutualism, Penicillium, plant protection.

\section{INTRODUCTION}

Endophytes are defined as organisms that colonize living, internal tissues of plants without causing any immediate, overt negative effect [1]. So close is the association with their hosts that they have been depicted to form an integral part of the 'extended phenotype' or symbiotic community of plant species $[2,3]$. Their occurrence in plant tissues may result upon the establishment of quite complex ecological and trophic relationships ranging from an adaptation of saprophytism also defined as 'balanced antagonism' [4], where the microbe remains quiescent at more or less circumscribed sites awaiting to colonize host tissues eventually damaged by biotic or abiotic stresses, to a true mutualism, established when the microbe is somehow allowed to spread within the host offering in turn some kind of ecological or physiological advantage. In a sense, such a variation in the kind of relationships makes the concept of endophytism purely topographical, and reflects the existence of a wide range of micro-organisms that are more or less adapted to this biocenotic condition, hence more or less regularly associated to a particular plant species.

Until recently, the role of microbes in the organization of plant communities has been neglected. However, improvements in the ability to isolate, identify, and monitor endophytes have enabled researchers to more thoroughly consider the complex ecological relationships with their hosts. Thus, it is now generally agreed that microbial endophytes interact with plants at genetic and physiological levels, and that these interactions substantially influence plant fitness, with potential effects on vegetation dynamics.

*Address correspondence to this author at the Consiglio per la Ricerca e la Sperimentazione in Agricoltura, Unità di Ricerca CAT, Via Vitiello 108, Scafati 84018, Italy; Tel: (39) 081-8563631; Fax: (39) 081-8506206;

E-mail: rosario.nicoletti@entecra.it
Virtually spread in every plant on earth, endophytic fungi are influenced in their occurrence and detection by a multiplicity of extrinsic and intrinsic factors, such as the phytogeographic context, the habitat and its microclimatic and anthropic modifications, the plant taxa, the age and specificity of the colonized tissue, the number of samples used for isolations, the timing of the sampling activities, the isolation media, etc. Figures are therefore pure guesswork, and the tentatively-estimated astonishing number of over one million species is much higher than all the fungal species currently known $[5,6]$. Within such a multitude, Penicillium represents one of the most commonly reported taxa, including species able to adapt to the most varied environmental conditions. Their well-known ability to produce a plethora of bioactive secondary metabolites has stimulated a multiplicity of research activities addressed to investigate both the involvement in plant protection based on a possible induction of antagonistic effects toward agents of biotic adversities, and the opportunities for applications in human medicine, considering the relevance of natural products for the development of novel antibiotic, immunosuppressant, and anticancer drugs. This paper offers an overview on the occurrence of endophytic Penicillium species in woody plants, with reference to their ecological role and implications in bioprospecting.

\section{TAXONOMIC CONTEXT}

Until recently Penicillium indicated the anamorphic stage of ascomycetous fungi belonging to the genera Eupenicillium and Talaromyces (Eurotiales, Trichocomaceae). Nevertheless, it traditionally prevailed in nomenclature by reason of a more general occurrence of the anamorph, which in most cases represents the form that can be isolated and cultured in the laboratory. A fundamental 
taxonomic revision based on a detailed phylogenetic analysis $[7,8]$ has brought to the separation of the biverticillate species (Penicillium subgenus Biverticillium) in Talaromyces, which has been kept in the Trichocomaceae. At the same time species in the other subgenera and in the former genera Chromocleista, Eladia, Hemicarpenteles, Thysanophora and Torulomyces, forming a single clade, have been placed in Penicillium sensu stricto, as well including the teleomorphic taxa formerly ascribed to Eupenicillium, and segregated to the family Aspergillaceae.

By reason of this rearrangement, species that have been repeatedly reported for their endophytic occurrence, such as Thysanophora penicillioides, are included in this review with their updated name (Table 1); conversely, species of Talaromyces are not considered, together with unclassified strains provisionally reported as Penicillium sp. whose morphological description conforms with the symmetric biverticillate condition [9].

Additional nomenclatural problems may derive from an increasing number of new reports and the examination by specialists of deposited strains, which occasionally disclose synonymy with other taxa deserving priority, or are even typified as novel species. Species treated in this paper are cited under their latest accepted denomination, which therefore does not necessarily correspond to the one used in the pertinent references. However, considering the ongoing taxonomic revision based on the application of biomolecular techniques, further changes in the species status of some taxa may not have timely considered in this manuscript.

The present review is also limited to strains/species which have been recovered after a surface disinfection of the plant samples intended to prevent the isolation of epiphytes. Therefore data based on isolations carried out through alternative methods, such as serial washings $[10,11]$, are not considered.

\section{ENDOPHYTIC PENICILLIUM SPECIES AND THEIR RELATIONSHIPS WITH HOST PLANTS}

\section{Phytopathological Implications}

According to the above definition, plant pathogens should not be included in the category of endophytes. However, many fungal pathogens present a latent stage which may account for their occurrence in symptomless plant organs [12], a reason why it is relevant to mention species which have been reported for such an aptitude. Disease caused by Penicillia on woody plants is particularly inherent to species known as agents of fruit rots, such as $P$. aurantiogriseum, $P$. commune, $P$. crustosum, $P$. digitatum, $P$. expansum, $P$. glabrum, $P$. implicatum, $P$. italicum, $P$. solitum and $P$. ulaiense, whose incidence is generally low in the field, but may dramatically increase after harvesting [13-19].

Additional concern for plant pathologists derives from the possible release of mycotoxins which may accumulate in plants and/or their edible products. The ability by $P$. expansum to produce patulin is particularly considered in apples, a few stone fruits and grapes, and the finding of this mycotoxin in symptomless fruits has raised concerns for a possible intake deriving from latent strains [20, 21]. To this regard no circumstantial evidence has resulted so far, despite a reported endophytic ability of this species (Table 1).

Likewise, a conjecture has been advanced that the presence of ochratoxin A in coffee derives by its accumulation before harvesting as a metabolic product of fungal endophytes. The occurrence of endophytic Penicillium species in Coffea spp. has been quite well documented, with the recovery of as many as 16 species from different plant parts and cultivation areas, together with additional unidentified isolates (Tables $\mathbf{1}$ and 2). Isolates of four Penicillium species were found to be able to produce just small quantities of ochratoxin A (Table 3 ), reflecting a negligible contribution to the presence of this mycotoxin in processed coffee [22]. However, similar investigations on additional mycotoxins known to be produced by species such as $P$. citrinum should be carried out for a more thorough assessment of the actual relevance of endophytic Penicillium strains in view of an eventual contamination of the processed product. It is quite meaningful to note that the research activity carried out on this particular subject has brought to the description of a new species, $P$. coffeae, after an isolation from a peduncle of a single plant of Coffea arabica grown at an experimental field in the island of Oahu [23]. Nevertheless, the intriguing assumption of this taxon as a Hawaiian endemism has been excluded by a novel isolation from sago starch in the Balima region of Papua New Guinea [24].

Possible implications in plant health may involve Penicillium spp., such as $P$. glabrum and $P$. paxilli, reported to occur in insect galls or cecidia [25]. However, these strains should not be regarded as endophytes in the strict sense, considering that galls are abnormal outgrowths originated after the aggression of a pest, and that the fungal presence may more directly result after the establishment of a saprophytic, entomopathogenic, or symbiotic relationship with the latter organism $[26,27]$.

\section{Mutualism}

Endophytes contribute to improve host fitness through a set of beneficial effects [3]. Particularly, the production of antimicrobial metabolites and free radical-scavenging substances may enhance host's tolerance to biotic and abiotic stresses, and the concept of 'defensive mutualism' has been defined to explain endophyte-mediated plant protection through the production of a range of bioactive molecules [28]. Moreover, an adaptative relationship referable to a specific function in plant protection has been conjectured for vertically transmitted endophytes [29]. As a matter of fact, incidence of culturable fungal endophytic biodiversity may be even more substantial than it could be optimistically hoped. A meaningful example is offered by an investigation carried out on cocoa (Theobroma cacao) showing that about $70 \%$ endophytic strains, including Penicillium spp., had some extent of biocontrol effects against the agent of the black-pod rot disease (Phytophthora palmivora) [30].

There are a number of additional reports considering Penicillium in antagonism against plant pathogens, and possible applications in biological control. Examples on woody 
Table 1. Penicillium species reported for endophytic occurrence.

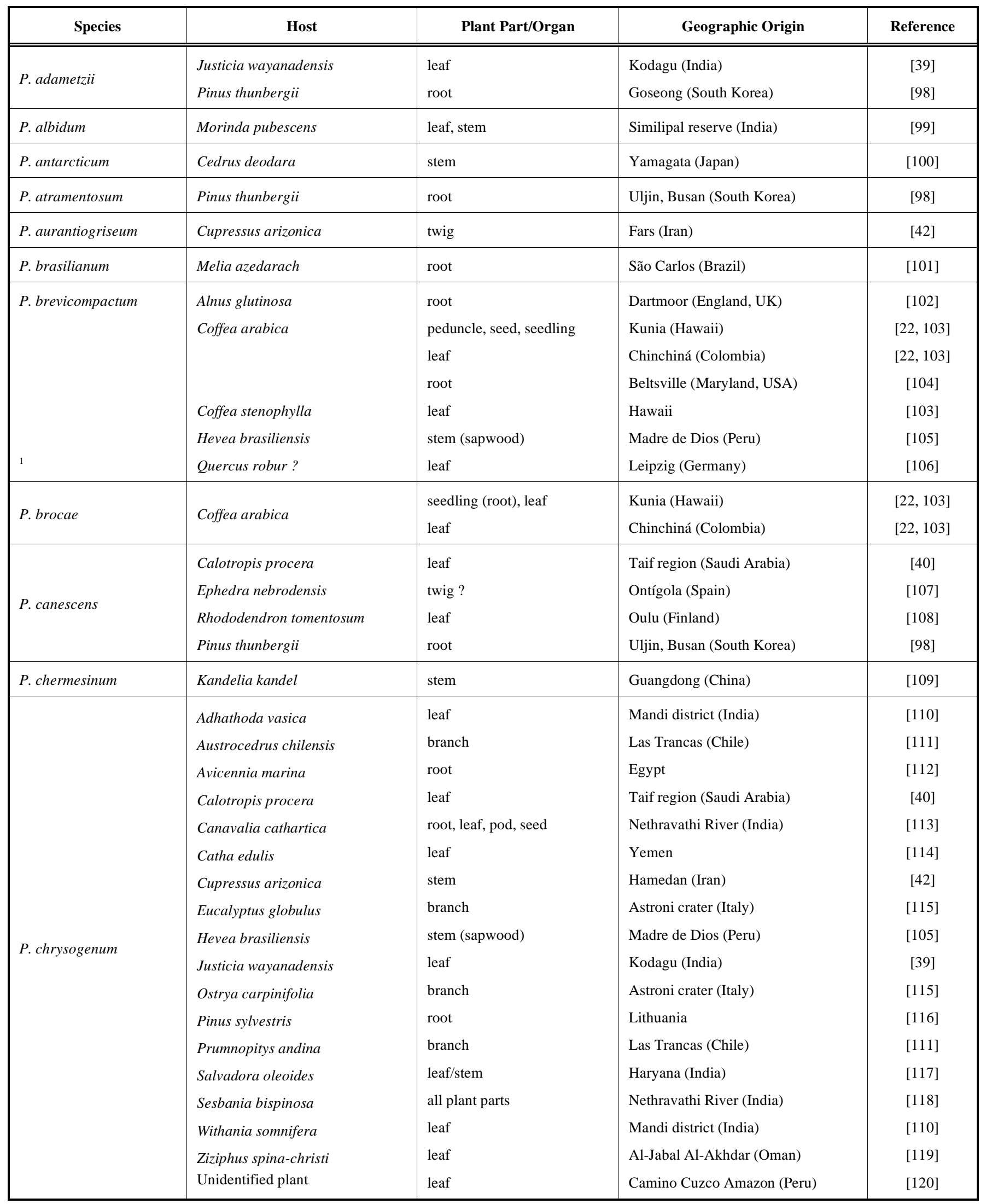


Table 1. contd....

\begin{tabular}{|c|c|c|c|c|c|}
\hline Species & & Host & Plant Part/Organ & Geographic Origin & Reference \\
\hline P. citreonigrum & & $\begin{array}{l}\text { Picea abies } \\
\text { Pinus thunbergii }\end{array}$ & $\begin{array}{l}\text { needle } \\
\text { root }\end{array}$ & $\begin{array}{l}\text { Lombardia (Italy) } \\
\text { Busan (South Korea) }\end{array}$ & $\begin{array}{c}{[121]} \\
{[98]}\end{array}$ \\
\hline P. citrinum & & $\begin{array}{l}\text { Abies beshanzuensis } \\
\text { Alnus glutinosa } \\
\text { Catha edulis } \\
\text { Ceratonia siliqua } \\
\text { Coffea arabica } \\
\text { Melia azedarach } \\
\text { Palicourea tetraphylla } \\
\text { Prosopis cineraria } \\
\text { Taxus cuspidata } \\
\text { Ziziphus hajnanensis } \\
\text { Ziziphus spina-christi }\end{array}$ & $\begin{array}{l}\text { needle or twig } \\
\text { root } \\
\text { leaf } \\
\text { stem } \\
\text { peduncle } \\
\text { seedling (root, leaf) } \\
\text { stem, leaf, root } \\
\text { stem, leaf (cortex) } \\
\text { leaf } \\
\text { inner bark } \\
\text { bark } \\
\text { leaf } \\
\text { leaf }\end{array}$ & $\begin{array}{l}\text { Baishanzu (China) } \\
\text { Dartmoor (England, UK) } \\
\text { Yemen } \\
\text { Morocco } \\
\text { Kunia (Hawaii) } \\
\text { Beltsville (Maryland, USA) } \\
\text { Beltsville (Maryland, USA) } \\
\text { Brazil } \\
\text { Serra do Espinhaço (Brazil) } \\
\text { India } \\
\text { Changbai Mountain (China) } \\
\text { Al-Jabal Al-Akhdar (Oman) } \\
\text { Al-Jabal Al-Akhdar (Oman) }\end{array}$ & $\begin{array}{c}{[3]} \\
{[102]} \\
{[114]} \\
{[64]} \\
{[22,103]} \\
{[22]} \\
{[104]} \\
{[122]} \\
{[123]} \\
{[124]} \\
{[125]} \\
{[119]} \\
{[119]}\end{array}$ \\
\hline P. coffeae & & Coffea arabica & peduncle & Kunia (Hawaii) & {$[23]$} \\
\hline P. commune & & $\begin{array}{l}\text { Cupressus arizonica } \\
\text { Espeletia sp. } \\
\text { Hibiscus tiliaceus } \\
\text { Olea europaea } \\
\text { Persea americana } \\
\text { Sorbus sp. }\end{array}$ & $\begin{array}{l}\text { stem } \\
\text { trunk? } \\
\text { stem } \\
\text { leaf or branch } \\
\text { root } \\
\text { not specified }\end{array}$ & $\begin{array}{l}\text { Fars, Hamedan, Markazi (Iran) } \\
\text { Choachí (Colombia) } \\
\text { Hainan (China) } \\
\text { Portugal } \\
\text { South Africa } \\
\text { not specified }\end{array}$ & $\begin{array}{c}{[42]} \\
{[126]} \\
{[127]} \\
{[41]} \\
{[128]} \\
{[129]}\end{array}$ \\
\hline P. cristata & $*$ & $\begin{array}{l}\text { Azadirachta indica } \\
\text { Eucalyptus citriodora }\end{array}$ & $\begin{array}{l}\text { root, fruit } \\
\text { leaf }\end{array}$ & $\begin{array}{l}\text { Varanasi (India) } \\
\text { Varanasi (India) }\end{array}$ & $\begin{array}{l}{[131]} \\
{[132]}\end{array}$ \\
\hline P. crustosum & & $\begin{array}{l}\text { Coffea arabica } \\
\text { Persea americana } \\
\text { Quercus robur }\end{array}$ & $\begin{array}{l}\text { seed } \\
\text { berry } \\
\text { crown, berry } \\
\text { seed } \\
\text { root } \\
\text { branch }\end{array}$ & $\begin{array}{l}\text { Cacaohoatán (Mexico) } \\
\text { Guatemala } \\
\text { Chinchiná (Colombia) } \\
\text { Águas da Prata (Brazil) } \\
\text { South Africa } \\
\text { Astroni crater (Italy) }\end{array}$ & $\begin{array}{c}{[22,103]} \\
{[133]} \\
{[103]} \\
{[134]} \\
{[128]} \\
{[115]}\end{array}$ \\
\hline P. daleae & $*$ & $\begin{array}{l}\text { Coffea arabica } \\
\text { Pinus thunbergii } \\
\text { Taxus chinensis }\end{array}$ & $\begin{array}{l}\text { stem, root } \\
\text { root } \\
\text { leaf }\end{array}$ & $\begin{array}{l}\text { Beltsville (Maryland, USA) } \\
\text { Goseong (South Korea) } \\
\text { Jinyun reserve (China) }\end{array}$ & $\begin{array}{c}{[104]} \\
{[98]} \\
{[135]}\end{array}$ \\
\hline P. dipodomyicola & & Palicourea tetraphylla & leaf & Serra do Espinhaço (Brazil) & [123] \\
\hline P. donkii & & Eucalyptus grandis & twig & Tacuarembó (Uruguay) & {$[136]$} \\
\hline P. echinulatum & & $\begin{array}{l}\text { Carpinus betulus } \\
\text { Cupressus arizonica } \\
\text { Cupressus sempervirens } \\
\text { Thuja orientalis }\end{array}$ & $\begin{array}{l}\text { branch } \\
\text { stem } \\
\text { twig } \\
\text { leaf }\end{array}$ & $\begin{array}{l}\text { Astroni crater (Italy) } \\
\text { Hamedan (Iran) } \\
\text { Fars (Iran) } \\
\text { Hamedan (Iran) }\end{array}$ & $\begin{array}{l}{[115]} \\
{[42]} \\
{[42]} \\
{[42]}\end{array}$ \\
\hline
\end{tabular}


Table 1. contd....

\begin{tabular}{|c|c|c|c|c|}
\hline Species & Host & Plant Part/Organ & Geographic Origin & Reference \\
\hline P. expansum & $\begin{array}{l}\text { Alnus nepalensis } \\
\text { Cupressus arizonica } \\
\text { Excoecaria agallocha } \\
\text { Picea abies } \\
\text { Sorbus sp. } \\
\text { Thuja orientalis }\end{array}$ & $\begin{array}{l}\text { leaf } \\
\text { leaf } \\
\text { root } \\
\text { needle } \\
\text { not specified } \\
\text { twig }\end{array}$ & $\begin{array}{l}\text { Taphou Naga (India) } \\
\text { Hamedan (Iran) } \\
\text { Wenchang (China) } \\
\text { Lombardia (Italy) } \\
\text { not specified } \\
\text { Fars (Iran) }\end{array}$ & $\begin{array}{c}{[137]} \\
{[42]} \\
{[138]} \\
{[121]} \\
{[129]} \\
{[42]}\end{array}$ \\
\hline P. fellutanum & Pinus rigida & needle & Daejeon (South Korea) & [139] \\
\hline P. freii $\quad *$ & Platycladus orientalis & foliage & North Carolina, USA & [140] \\
\hline P. glabrum & $\begin{array}{l}\text { Acer saccharum } \\
\text { Coffea arabica } \\
\text { Espeletia sp. } \\
\text { Eucalyptus nitens } \\
\text { Hevea brasiliensis } \\
\text { Manilkara bidentata } \\
\text { Pinus thunbergii } \\
\text { Punica granatum } \\
\text { Quercus ilex } \\
\text { Theobroma gileri }\end{array}$ & $\begin{array}{l}\text { leaf (lamina, petiol) } \\
\text { root } \\
\text { leaf } \\
\text { twig } \\
\text { leaf } \\
\text { leaf } \\
\text { root } \\
\text { fruit } \\
\text { bark, twig and/or leaf } \\
\text { leaf or twig } \\
\text { bole (sapwood) }\end{array}$ & $\begin{array}{l}\text { Southern Quebec (Canada) } \\
\text { Beltsville (Maryland, USA) } \\
\text { Choachí (Colombia) } \\
\text { Canberra (Australia) } \\
\text { Madre de Dios (Peru) } \\
\text { Luquillo (Puerto Rico) } \\
\text { Uljin, Busan (South Korea) } \\
\text { Uzbekistan } \\
\text { Castilla (Spain) } \\
\text { unspecified } \\
\text { Esmeraldas (Ecuador) }\end{array}$ & $\begin{array}{l}{[141]} \\
{[104]} \\
{[142]} \\
{[143]} \\
{[105]} \\
{[144]} \\
{[98]} \\
{[63]} \\
{[145]} \\
{[146]} \\
{[147]}\end{array}$ \\
\hline P. glaucoalbidum ${ }^{2}$ & $\begin{array}{l}\text { Abies alba } \\
\text { Abies beshanzuensis } \\
\text { Fagus sylvatica } \\
\text { Picea abies } \\
\text { Picea glauca }\end{array}$ & $\begin{array}{l}\text { needle } \\
\text { needle } \\
\text { leaf } \\
\text { needle } \\
\text { needle } \\
\text { needle }\end{array}$ & $\begin{array}{l}\text { Switzerland } \\
\text { Baishanzu Reserve (China) } \\
\text { Greifswald (Germany) } \\
\text { Southern Finland } \\
\text { Trojmezná (Czech Rep.) } \\
\text { Southern Québec (Canada) }\end{array}$ & $\begin{array}{l}{[148]} \\
{[38]} \\
{[149]} \\
{[150]} \\
{[151]} \\
{[152]}\end{array}$ \\
\hline P. goetzii & $\begin{array}{l}\text { Pinus ponderosa } \\
\text { Pseudotsuga menziesii }\end{array}$ & $\begin{array}{l}\text { root } \\
\text { root }\end{array}$ & $\begin{array}{l}\text { Okanogan (Washington, USA) } \\
\text { Okanogan (Washington, USA) }\end{array}$ & $\begin{array}{l}{[153]} \\
{[153]}\end{array}$ \\
\hline P. griseofulvum & Palicourea tetraphylla & leaf & Serra do Espinhaço (Brazil) & [123] \\
\hline P. griseoroseum & $\begin{array}{l}\text { Coffea arabica } \\
\text { Picea abies }\end{array}$ & $\begin{array}{l}\text { seed } \\
\text { needle }\end{array}$ & $\begin{array}{l}\text { Aguas da Prata (Brazil) } \\
\text { Lombardia (Italy) }\end{array}$ & $\begin{array}{c}{[79]} \\
{[121]}\end{array}$ \\
\hline P. hennebertii ${ }^{3}$ & Picea abies & needle & Lombardia (Italy) & [121] \\
\hline P. herquei & Melia azedarach & fruit & Brazil & [122] \\
\hline P. implicatum & Melia azedarach & fruit & Brazil & {$[122]$} \\
\hline P. italicum & $\begin{array}{l}\text { Alnus nepalensis } \\
\text { Canavalia cathartica }\end{array}$ & $\begin{array}{l}\text { leaf } \\
\text { root, pod }\end{array}$ & $\begin{array}{l}\text { Taphou Naga (India) } \\
\text { Nethravathi River (India) }\end{array}$ & $\begin{array}{l}{[137]} \\
{[113]}\end{array}$ \\
\hline P. janczewskii & Prumnopitys andina & branch (phloem) & Las Trancas (Chile) & {$[111,154]$} \\
\hline P. janthinellum & $\begin{array}{l}\text { Coffea arabica } \\
\text { Melia azedarach } \\
\text { Pinus thunbergii } \\
\text { Taxus baccata } \\
\text { unspecified mangrove }\end{array}$ & $\begin{array}{l}\text { root } \\
\text { root } \\
\text { fruit } \\
\text { root } \\
\text { inner bark } \\
\text { leaf }\end{array}$ & $\begin{array}{l}\text { Kunia (Hawaii) } \\
\text { Beltsville (Maryland, USA) } \\
\text { Brazil } \\
\text { Busan (South Korea) } \\
\text { Arunachal Pradesh (India) } \\
\text { Karankadu (India) }\end{array}$ & $\begin{array}{c}{[22]} \\
{[104]} \\
{[122]} \\
{[98]} \\
{[155]} \\
{[85]}\end{array}$ \\
\hline
\end{tabular}


Table 1. contd....

\begin{tabular}{|c|c|c|c|c|}
\hline Species & Host & Plant Part/Organ & Geographic Origin & Reference \\
\hline P. lagena ${ }^{4}$ & $\begin{array}{l}\text { Bauhinia racemosa } \\
\text { Diospyros montana } \\
\text { Elaeodendron glaucum } \\
\text { Erica arborea } \\
\text { Ixora nigricans } \\
\text { Quercus ilex }\end{array}$ & $\begin{array}{l}\text { leaf } \\
\text { leaf } \\
\text { leaf } \\
\text { root } \\
\text { leaf } \\
\text { root }\end{array}$ & $\begin{array}{l}\text { Nilgiri mountains (India) } \\
\text { Nilgiri mountains (India) } \\
\text { Nilgiri mountains (India) } \\
\text { Liguria (Italy) } \\
\text { Nilgiri mountains (India) } \\
\text { Liguria (Italy) }\end{array}$ & $\begin{array}{l}{[156]} \\
{[156]} \\
{[156]} \\
{[157]} \\
{[156]} \\
{[157]}\end{array}$ \\
\hline P. levitum & Avicennia marina & root & Egypt & [112] \\
\hline P. meleagrinum & Hevea brasiliensis & leaf & Madre de Dios (Peru) & {$[105]$} \\
\hline P. multicolor & Abies beshanzuensis & needle or twig & Baishanzu (China) & {$[3]$} \\
\hline P. miczynskii & Taxus chinensis & bark & Jinyun reserve (China) & {$[158]$} \\
\hline P. montanense & Pinus thunbergii & root & Goseong (South Korea) & [98] \\
\hline P. nodositatum & $\begin{array}{l}\text { Alnus cordata } \\
\text { Alnus glutinosa } \\
\text { Alnus incana } \\
\text { Alnus viridis } \\
\text { Linnea borealis }\end{array}$ & $\begin{array}{l}\text { root } \\
\text { root } \\
\text { root } \\
\text { root } \\
\text { root } \\
\text { root } \\
\text { leaf }\end{array}$ & $\begin{array}{l}\text { Santabarbara (Italy) } \\
\text { Saou (France), Firenze (Italy) } \\
\text { Dauphiné (France) } \\
\text { Holland } \\
\text { Ardenne, Savoie, Dauphiné (France) } \\
\text { Savoie, Dauphiné (France) } \\
\text { Oregon, USA }\end{array}$ & $\begin{array}{c}{[159]} \\
{[70]} \\
{[68,70]} \\
{[72]} \\
{[68-71]} \\
{[70]} \\
{[73]}\end{array}$ \\
\hline P. ochrochloron & Pinus thunbergii & root & Busan (South Korea) & [98] \\
\hline P. olsonii & $\begin{array}{l}\text { Atriplex canescens } \\
\text { Coffea arabica } \\
\text { Coffea congensis } \\
\text { Coffea dewevrei } \\
\text { Coffea liberica } \\
\text { Picea abies }\end{array}$ & $\begin{array}{l}\text { seed } \\
\text { crown, seed, berry, leaf } \\
\text { berry, stem } \\
\text { crown, berry } \\
\text { peduncle } \\
\text { leaves } \\
\text { peduncle, leaf } \\
\text { needle }\end{array}$ & $\begin{array}{l}\text { Las Cruces (New Mexico, USA) } \\
\text { Kauai, Kona, Kunia, Oahu (Hawaii) } \\
\text { Chinchiná (Colombia) } \\
\text { Puerto Rico } \\
\text { Kona (Hawaii) } \\
\text { Kona (Hawaii) } \\
\text { Kona (Hawaii) } \\
\text { Lombardia (Italy) }\end{array}$ & $\begin{array}{c}{[160]} \\
{[22,103]} \\
{[22,103]} \\
{[103]} \\
{[22]} \\
{[22]} \\
{[22,103]} \\
{[121]}\end{array}$ \\
\hline P. oxalicum & $\begin{array}{l}\text { Abies beshanzuensis } \\
\text { Coffea arabica } \\
\text { Theobroma cacao } \\
\text { Thuja plicata }\end{array}$ & $\begin{array}{l}\text { needle or twig } \\
\text { leaf } \\
\text { seed } \\
\text { leaf, stem }\end{array}$ & $\begin{array}{l}\text { Baishanzu (China) } \\
\text { Chinchiná (Colombia) } \\
\text { Beltsville (Maryland, USA) } \\
\text { Chennai (India) }\end{array}$ & $\begin{array}{c}{[3]} \\
{[22,103]} \\
{[161]} \\
{[162]}\end{array}$ \\
\hline P. parvum & Azadirachta indica & not specified & Varanasi district (India) & [47] \\
\hline P. paxilli & $\begin{array}{l}\text { Garcinia atroviridis } \\
\text { Hevea brasiliensis }\end{array}$ & $\begin{array}{l}\text { leaf or branch } \\
\text { stem (sapwood), leaf }\end{array}$ & $\begin{array}{l}\text { Southern Thailand } \\
\text { Madre de Dios (Peru) }\end{array}$ & $\begin{array}{l}{[163]} \\
{[105]}\end{array}$ \\
\hline P. polonicum & Lysidice rhodostegia & root & China & [164] \\
\hline P. raciborskii & Rhododendron tomentosum & leaf & Oulu (Finland) & {$[108]$} \\
\hline P. raistrickii & $\begin{array}{l}\text { Pinus thunbergii } \\
\text { Taxus brevifolia }\end{array}$ & $\begin{array}{l}\text { root } \\
\text { inner bark }\end{array}$ & $\begin{array}{l}\text { Busan (South Korea) } \\
\text { Northwestern Montana (USA) }\end{array}$ & $\begin{array}{c}{[98]} \\
{[165]}\end{array}$ \\
\hline
\end{tabular}


Table 1. contd....

\begin{tabular}{|c|c|c|c|c|}
\hline Species & Host & Plant Part/Organ & Geographic Origin & Reference \\
\hline P. cf. resedanum & Tilia cordata? & leaf & Leipzig (Germany) & [106] \\
\hline P. rolfsii & Pinus thunbergii & root & Uljin, Busan (South Korea) & [98] \\
\hline P. roseopurpureum * & $\begin{array}{l}\text { Coffea arabica } \\
\text { Olea europaea } \\
\text { Pinus thunbergii }\end{array}$ & $\begin{array}{l}\text { seed } \\
\text { leaf or branch } \\
\text { root }\end{array}$ & $\begin{array}{l}\text { Kunia (Hawaii) } \\
\text { Portugal } \\
\text { Busan (South Korea) }\end{array}$ & $\begin{array}{l}{[22]} \\
{[41]} \\
{[98]}\end{array}$ \\
\hline P. sclerotiorum & $\begin{array}{l}\text { Abies beshanzuensis } \\
\text { Alchornea castaneifolia } \\
\text { Camellia sinensis } \\
\text { Coffea arabica } \\
\text { Eugenia aff. bimarginata } \\
\text { Garcinia atroviridis } \\
\text { Hevea brasiliensis } \\
\text { Taxus chinensis }\end{array}$ & $\begin{array}{l}\text { needle or twig } \\
\text { leaf } \\
\text { leaf, stem } \\
\text { crown, peduncle } \\
\text { leaf } \\
\text { leaf } \\
\text { stem (sapwood) } \\
\text { branch }\end{array}$ & $\begin{array}{l}\text { Baishanzu (China) } \\
\text { Tocantins (Brazil) } \\
\text { Zijin hill (China) } \\
\text { Kauai (Hawaii) } \\
\text { Tocantins (Brazil) } \\
\text { Yala (Thailand) } \\
\text { Madre de Dios (Peru) } \\
\text { Jinggang mountains (China) }\end{array}$ & $\begin{array}{c}{[3]} \\
{[166]} \\
{[167]} \\
{[22,103]} \\
{[166]} \\
{[168]} \\
{[105]} \\
{[158]}\end{array}$ \\
\hline P. senticosum & unspecified medicinal plant & leaf & Tirumala hills (India) & {$[86]$} \\
\hline P. simplicissimum & $\begin{array}{l}\text { Alnus glutinosa } \\
\text { Eucalyptus nitens } \\
\text { Melia azedarach }\end{array}$ & $\begin{array}{l}\text { root } \\
\text { twig } \\
\text { stem (cortex) }\end{array}$ & $\begin{array}{l}\text { Dartmoor (England, UK) } \\
\text { Canberra (Australia) } \\
\text { Brazil }\end{array}$ & $\begin{array}{l}{[102]} \\
{[143]} \\
{[122]}\end{array}$ \\
\hline P. spinulosum & $\begin{array}{l}\text { Eucalyptus nitens } \\
\text { Hevea brasiliensis } \\
\text { Picea glauca } \\
\text { Taxus chinensis }\end{array}$ & $\begin{array}{l}\text { not specified } \\
\text { stem (sapwood) } \\
\text { needle } \\
\text { bark }\end{array}$ & $\begin{array}{l}\text { not specified } \\
\text { Madre de Dios (Peru) } \\
\text { New Brunswick (Canada) } \\
\text { Jinyun reserve (China) }\end{array}$ & $\begin{array}{l}{[143]} \\
{[105]} \\
{[43]} \\
{[158]}\end{array}$ \\
\hline P. steckii & Coffea arabica & $\begin{array}{l}\text { berry } \\
\text { root }\end{array}$ & $\begin{array}{l}\text { Oahu (Hawaii) } \\
\text { Beltsville (Maryland, USA) }\end{array}$ & $\begin{array}{l}{[22,103]} \\
{[22,104]}\end{array}$ \\
\hline P. sublateritium & Calotropis procera & stem & Karachi (Pakistan) & [169] \\
\hline P. sumatrense & $\begin{array}{l}\text { Abies beshanzuensis } \\
\text { Coffea arabica } \\
\text { Vitis vinifera }\end{array}$ & $\begin{array}{l}\text { needle or twig } \\
\text { peduncle } \\
\text { seed } \\
\text { not specified }\end{array}$ & $\begin{array}{l}\text { Baishanzu (China) } \\
\text { Chiapas (Mexico) } \\
\text { Adjuntas (Puerto Rico) } \\
\text { Fujian (China)? }\end{array}$ & $\begin{array}{c}{[3]} \\
{[103]} \\
{[103]} \\
{[170]}\end{array}$ \\
\hline P. thomii & $\begin{array}{l}\text { Alnus glutinosa } \\
\text { Bruguiera gymnorrhiza } \\
\text { Picea glauca } \\
\text { Terminalia chebula }\end{array}$ & $\begin{array}{l}\text { root } \\
\text { root } \\
\text { needle } \\
\text { leaf, stem or root }\end{array}$ & $\begin{array}{l}\text { Dartmoor (England, UK) } \\
\text { Guang Xi (China) } \\
\text { New Brunswick (Canada) } \\
\text { Dhaka (Bangladesh) }\end{array}$ & $\begin{array}{l}{[102]} \\
{[171]} \\
{[43]} \\
{[172]}\end{array}$ \\
\hline P. toxicarium & $\begin{array}{l}\text { Coffea arabica } \\
\text { Pinus rigida }\end{array}$ & $\begin{array}{l}\text { stem } \\
\text { needle }\end{array}$ & $\begin{array}{l}\text { Beltsville (Maryland, USA) } \\
\text { Daejeon (South Korea) }\end{array}$ & $\begin{array}{l}{[104]} \\
{[139]}\end{array}$ \\
\hline P. verrucosum & Quercus robur or Q. cerris & leaf, shoot, twig or bud & Trino (Italy) & [173] \\
\hline P. viridicatum & Cupressus sempervirens & twig & Fars (Iran) & [42] \\
\hline P. waksmanii & Alnus glutinosa & root & Dartmoor (England, UK) & {$[102]$} \\
\hline
\end{tabular}

${ }^{1}$ strain identified as $P$. cf. biourgeianum $;{ }^{2}$ reported as the synonym Thysanophora penicillioides $;{ }^{3}$ reported as the synonym Thysanophora canadensis;

${ }^{4}$ reported as the synonym Torulomyces lagena; $*$ species identification on the corresponding host is questionable;

? host, plant part or location not clearly stated in the pertinent reference. 
Table 2. Occurrence of unclassified endophytic Penicillium strains.

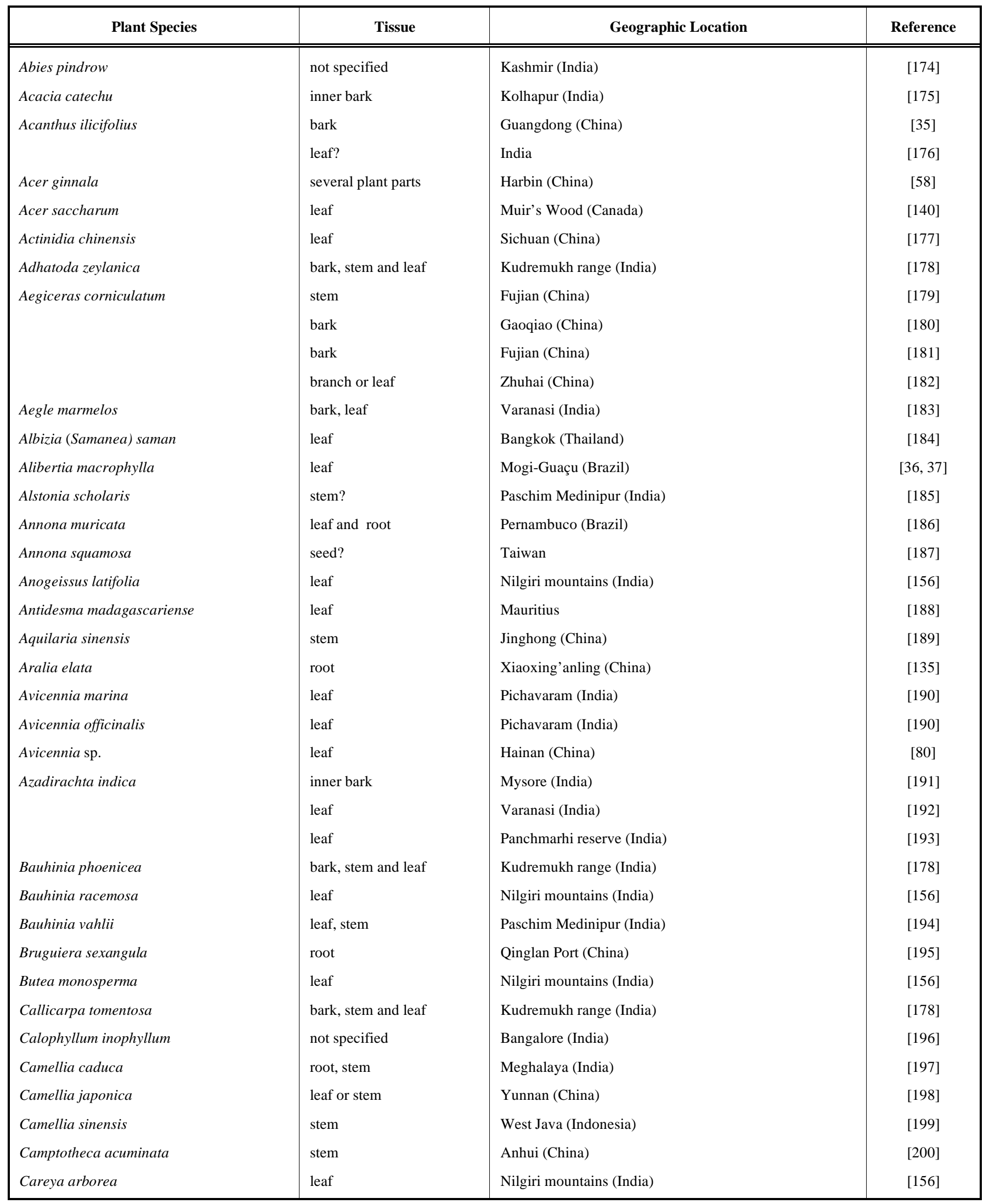


Table 2. contd...

\begin{tabular}{|c|c|c|c|}
\hline Plant Species & Tissue & Geographic Location & Reference \\
\hline Cassia fistula & leaf & Nilgiri mountains (India) & {$[156]$} \\
\hline Casuarina equisetifolia & shoot & Puerto Rico & [201] \\
\hline Cerbera manghas & stem? & China & {$[202]$} \\
\hline Ceriops tagal & leaf? & China & [203] \\
\hline Cinnamomum longepaniculatum & leaf & Yibin (China) & [204] \\
\hline Clerodendrum serratum & bark, stem and leaf & Kudremukh range (India) & {$[178]$} \\
\hline \multirow[t]{3}{*}{ Coffea arabica } & leaf & Puerto Rico & [205] \\
\hline & bean & Cundinamarca, Valle del Cauca (Colombia) & [206] \\
\hline & root & Ethiopia & [207] \\
\hline Cordemoya integrifolia & leaf & Maccabhé (Mauritius) & [208] \\
\hline Dalbergia oliveri & leaf & Viengsa (Thailand) & [209] \\
\hline Daphniphyllum longeracemosum & branch & Kunming (China) & [210] \\
\hline Delonix regia & leaf or stem & Yunnan (China) & {$[198]$} \\
\hline Derris elliptica & root & Guangzhou (China) & {$[55]$} \\
\hline Derris hancei & leaf, caudex & China & {$[56]$} \\
\hline Diospyros crassifolia & fruit & Mbalmayo (Cameroon) & [211] \\
\hline Dracaena cambodiana & stem & Yunnan (China) & [189] \\
\hline Elaeodendron glaucum & leaf & Nilgiri mountains (India) & {$[156]$} \\
\hline Eucalyptus benthami & seedling & Southern Brazil & {$[212]$} \\
\hline \multirow[t]{2}{*}{ Eucalyptus globulus } & stem & Maldonado (Uruguay) & [213] \\
\hline & leaf & La Coruña province (Spain) & [214] \\
\hline Eucalyptus grandis & twig & Tacuarembò (Uruguay) & [136] \\
\hline Fagus sylvatica & leaf & Greifswald (Germany) & {$[215]$} \\
\hline Garcinia atroviridis & leaf or branch & Southern Thailand & {$[163]$} \\
\hline \multirow[t]{2}{*}{ Ginkgo biloba } & bark & China & [216] \\
\hline & root & China & [217] \\
\hline Givotia rottleriformis & leaf & Nilgiri mountains (India) & {$[156]$} \\
\hline Glochidion ferdinandi & bark & Toohey Forest (Australia) & [218] \\
\hline Grewia tiliaefolia & leaf & Nilgiri mountains (India) & {$[156]$} \\
\hline Helicteres isora & leaf & Nilgiri mountains (India) & [156] \\
\hline Hopea hainanensis & leaf & Hainan (China) & {$[34]$} \\
\hline Ixora nigricans & leaf & Nilgiri mountains (India) & {$[156]$} \\
\hline Jatropha curcas & stem & China & [219] \\
\hline \multirow[t]{3}{*}{ Kandelia candel } & root & Hong Kong (China) & {$[220]$} \\
\hline & branch or leaf & Zhuhai (China) & [182] \\
\hline & leaf & Shankou (China) & [221] \\
\hline Kigelia pinnata & leaf & Chengalpattu (India) & {$[222]$} \\
\hline Lagerstroemia microcarpa & leaf & Nilgiri mountains (India) & {$[156]$} \\
\hline Leptospermum scoparium & leaf & Northland and Coromandel (New Zealand) & [223] \\
\hline Leucopogon parviflorus & root & New South Wales (Australia) & [224] \\
\hline
\end{tabular}


Table 2. contd...

\begin{tabular}{|c|c|c|c|}
\hline Plant Species & Tissue & Geographic Location & Reference \\
\hline Lithocarpus (Pasania) edulis & leaf & Kagoshima (Japan), Pichavaram (India) & [225] \\
\hline Lumnitzera racemosa & leaf & Puerto Rico & [190] \\
\hline Madhuca indica & leaf, bark & Uttar Pradesh (India) & [226] \\
\hline Manilkara bidentata & seed & São Carlos (Brazil) & {$[201]$} \\
\hline Mauritia flexиosa & root & Manaus (Brazil) & [227] \\
\hline \multirow[t]{2}{*}{ Melia azedarach } & root & Brazil & {$[78]$} \\
\hline & leaf, root cortex & Brazil & [122] \\
\hline Mitragyna javanica & leaf & Ayuthaya province (Thailand) & [228] \\
\hline Moringa oleifera & leaf & Yercaud forest (India) & [229] \\
\hline Morus alba & leaf & China & [230] \\
\hline Murraya paniculata & leaf & São Carlos (Brazil) & {$[54]$} \\
\hline Naringi crenulata & leaf & Nilgiri mountains (India) & {$[156]$} \\
\hline Nothapodytes foetida & leaf, stem, seed, fruit & Agumbe forest (India) & [231] \\
\hline Nyctanthes arbor-tristis & leaf, stem & Varanasi (India) & [232] \\
\hline Phoradendron perrottettii & needles & Lavras (Brazil) & {$[233]$} \\
\hline Picea abies & root & Lombardia (Italy) & [121] \\
\hline Pinus ponderosa & needles, twigs & Mission Creek (Washington, USA) & [234] \\
\hline Pinus roxburghii & not specified & Kashmir (India) & {$[174]$} \\
\hline Pinus spp. & needles & Palencia province (Spain) & [235] \\
\hline \multirow[t]{2}{*}{ Pinus sylvestris } & sapwood & Harjavalta (Finland) & [236] \\
\hline & leaf & Western Alps (Italy) & {$[237]$} \\
\hline Pinus thunbergii & root & South Korea & [98] \\
\hline Plumeria rubra & leaf, twig, bark & Chennai (India) & [238] \\
\hline Populus angustifolia & leaf & Weber river (Utah, USA) & [239] \\
\hline Populus euphratica & leaf & Tarim River basin (China) & [240] \\
\hline \multirow[t]{2}{*}{ Populus tremula } & leaf & Palencia province (Spain) & {$[241]$} \\
\hline & leaf & Umeå (Sweden) & [242] \\
\hline Premna tomentosa & bark & Nilgiri mountains (India) & {$[156]$} \\
\hline Prosopis cineraria & root & Rajahstan (India) & [124] \\
\hline Pseudotsuga menziesii & buds, leaves, twigs & Mission Creek (Washington, USA) & [234] \\
\hline Quercus cerris & bark or leaf & Southern Italy & [243] \\
\hline \multirow[t]{2}{*}{ Quercus ilex } & branch or twig & La Matilla (Spain) & {$[145]$} \\
\hline & stem, root & Castel Fusano (Italy) & [244] \\
\hline Quercus pannosa & leaf, stem & Baima Snow Mountain reserve (China) & {$[245]$} \\
\hline Quercus pubescens & sapwood, heartwood & Southern Italy & {$[243]$} \\
\hline Quercus robur & leaf or branch & Westerwald (Germany) & {$[246]$} \\
\hline Quercus spinosa & leaf, stem & Baima Snow Mountain reserve (China) & {$[245]$} \\
\hline Quercus suber & not specified & Bortigiadas (Sardinia, Italy) & [247] \\
\hline Quercus variabilis & leaf & Zijin Mountain (China) & [248] \\
\hline Rauwolfia serpentina & leaf, stem & Northeast India & [249] \\
\hline
\end{tabular}


Table 2. contd...

\begin{tabular}{|c|c|c|c|}
\hline Plant Species & Tissue & Geographic Location & Reference \\
\hline Rhizophora annamalayana & leaf & Vellar estuary (India) & {$[250]$} \\
\hline Rhizophora apiculata & stem, root & Pichavaram (India) & [251] \\
\hline Rhizophora mucronata & stem & Porong river (Java, Indonesia) & {$[252]$} \\
\hline Rhododendron anthopogon & root & Kalchuman lake (Nepal) & [253] \\
\hline Rhododendron spp. & leaf, stem & Baima Snow Mountain reserve (China) & [245] \\
\hline Rosa hybrida & leaf or stem & Yunnan (China) & [198] \\
\hline Santalum album & root & Guangdong province (China) & [254] \\
\hline Schima khasiana & root, stem & Meghalaya (India) & [197] \\
\hline Scurrula atropurpurea & leaf & Puncak Pass (Java, Indonesia) & [255] \\
\hline Shorea obtusa & leaf & Viengsa (Thailand) & [209] \\
\hline Shorea siamensis & branch or leaf & Viengsa (Thailand) & [209] \\
\hline Sonneratia caseolaris & leaf & Zhuhai (China) & [182] \\
\hline Stereospermum angustifolium & leaf & Nilgiri mountains (India) & {$[156]$} \\
\hline Strychnos potatorum & leaf, stem & Nilgiri mountains (India) & {$[156]$} \\
\hline Tabebuia argentea & leaf, stem, root & Karnataka (India) & {$[256]$} \\
\hline Tamarix chinensis & leaf & Laizhou Bay (China) & {$[52]$} \\
\hline Tapirira guianensis & leaf, shoot or twig & Lavras (Brazil) & [233] \\
\hline \multirow[t]{2}{*}{ Taxus baccata } & inner bark & Italy & [257] \\
\hline & inner bark & Arunachal Pradesh (India) & {$[155]$} \\
\hline Taxus brevifolia & several plant parts & Northwestern USA & {$[75,81]$} \\
\hline Taxus chinensis & branch, bark & Jingning, Jinyun reserve (China) & {$[158]$} \\
\hline Taxus cuspidata & leaf & Gunma prefecture (Japan) & [258] \\
\hline \multirow[t]{2}{*}{ Taxus globosa } & bark & Sierra Alta Hidalguense (Mexico) & [259] \\
\hline & leaf & Sierra Gorda Reserve (Mexico) & {$[76]$} \\
\hline Tectona grandis & inner bark, twig & Bangkok (Thailand) & [184] \\
\hline Terminalia arjuna & leaf & Karnataka (India) & {$[260]$} \\
\hline Terminalia crenulata & bole (sapwood) & Nilgiri mountains (India) & {$[156]$} \\
\hline Theobroma gileri & xylem and/or shoot & Pichincha (Ecuador) & {$[147]$} \\
\hline Viscum album & leaf & DC Bungalow (India) & {$[261]$} \\
\hline Vismia latifolia & leaf & French Guiana & {$[262]$} \\
\hline \multirow[t]{3}{*}{ Vitis vinifera } & leaf & Austria & [263] \\
\hline & leaf, twig, berry & Madrid region (Spain) & {$[264]$} \\
\hline & leaf & Tenerife (Spain) & [265] \\
\hline Withania somnifera & leaf & Karachi (Pakistan) & {$[130]$} \\
\hline Ziziphus hajnanensis & leaf & Al-Jabal Al-Akhdar (Oman) & [119] \\
\hline Ziziphus spina-christi & leaf & Al-Jabal Al-Akhdar (Oman) & [119] \\
\hline Ziziphus xylopyrus & leaf & Nilgiri mountains (India) & {$[156]$} \\
\hline Unspecified medicinal plants & & Malnad (India) & {$[83]$} \\
\hline
\end{tabular}

? host, plant part or location not clearly stated in the pertinent reference. 
Table 3. Secondary metabolites produced by endophytic Penicillium strains.

\begin{tabular}{|c|c|c|c|}
\hline Compound & Species & Reference & Bioactivity \\
\hline Altenuene, hydroxyaltenuene, epialtenuene & Penicillium sp. & [203] & $\#$ \\
\hline Antarone A-B* & P. antarcticum & [100] & \# \\
\hline Arisugacin $\mathrm{B}, \mathrm{F}, \mathrm{G}, \mathrm{I}^{*}, \mathrm{~J}^{*}$ & Penicillium sp. & {$[52,221]$} & $\mathrm{C}, \mathrm{E}$ \\
\hline Arugosin $I^{*}$ & Penicillium sp. & {$[181]$} & $\#$ \\
\hline Asperfumoid & Penicillium sp. & {$[34]$} & $\mathrm{C}, \mathrm{F}$ \\
\hline Asperphenamate & P. raistrickii & [81] & \# \\
\hline Asterric acid & P. glabrum & {$[63]$} & $\#$ \\
\hline $\begin{array}{l}\text { Austins: austinolide*, austinoneol*, dehydroaustin, acetoxydehydroaustin, neoaustin, } \\
\text { isoaustinone*, preaustinoids* }\end{array}$ & P. brasilianum & {$[78,101,266-268]$} & B \\
\hline Azadirachtin A-B & P. parvum & [47] & $\#$ \\
\hline Baccatin III, deacetylbaccatin III & P. raistrickii & {$[81]$} & $\#$ \\
\hline Bacillosporin A, C & Penicillium sp. & [181] & $\#$ \\
\hline Benzomalvin $\mathrm{B}, \mathrm{C}$ & $\begin{array}{c}\text { Penicillium sp. } \\
\text { P. raistrickii }\end{array}$ & [81] & \# \\
\hline Bile acids (cholic, deoxycholic, glycocholic, glycodeoxycholic) & Penicillium sp. & [255] & $\#$ \\
\hline Brasiliamide A, B, F* & P. brasilianum & {$[101]$} & B \\
\hline$\beta$-Carbolines & Penicillium sp. & [269] & \# \\
\hline Chermesinone A-C* & P. chermesinum & [109] & $\#$ \\
\hline $\begin{array}{l}\text { Chromans: } \\
\text { Acetyldihydroxyhydroxypropyldimethylchroman*, } \\
\text { acetyl-dihydroxypropylidenehydroxymethylchromanone* }\end{array}$ & Penicillium sp. & {$[210]$} & \# \\
\hline Hydroxymethoxydimethylisochroman, arohynapene D, citriquinochroman* & P. citrinum & {$[64]$} & $\mathrm{C}$ \\
\hline Chromone & Penicillium sp. & [269] & \# \\
\hline Cinchona alkaloids: cinchonin, cinchonidine, quinine, quinidine & Penicillium sp. & [77] & \# \\
\hline Citreoviridin & Penicillium sp. & [81] & S \\
\hline Citrinamide A & P. citrinum & {$[64]$} & \# \\
\hline Citrinin & $\begin{array}{c}\text { P. herquei } \\
\text { P. janthinellum } \\
\text { Penicillium sp. } \\
\text { Penicillium sp. } \\
\text { Penicillium sp. }\end{array}$ & $\begin{array}{l}{[270]} \\
{[271]} \\
{[272]} \\
{[180]} \\
{[220]}\end{array}$ & $\begin{array}{c}\# \\
\mathrm{~B}, \mathrm{P} \\
\mathrm{B} \\
\# \\
\mathrm{C}\end{array}$ \\
\hline Citrinin $\mathrm{H}-1$ & P. herquei & [272] & B \\
\hline Citrorosein & $\begin{array}{l}\text { P. janthinellum } \\
\text { P. herquei }\end{array}$ & $\begin{array}{c}{[271]} \\
{[270,272]}\end{array}$ & $\begin{array}{l}\# \\
\text { B }\end{array}$ \\
\hline Clavatol & $\begin{array}{c}\text { P. commune } \\
\text { P. griseoroseum }\end{array}$ & $\begin{array}{c}{[127]} \\
{[79]}\end{array}$ & \# \\
\hline
\end{tabular}


Table 3. contd...

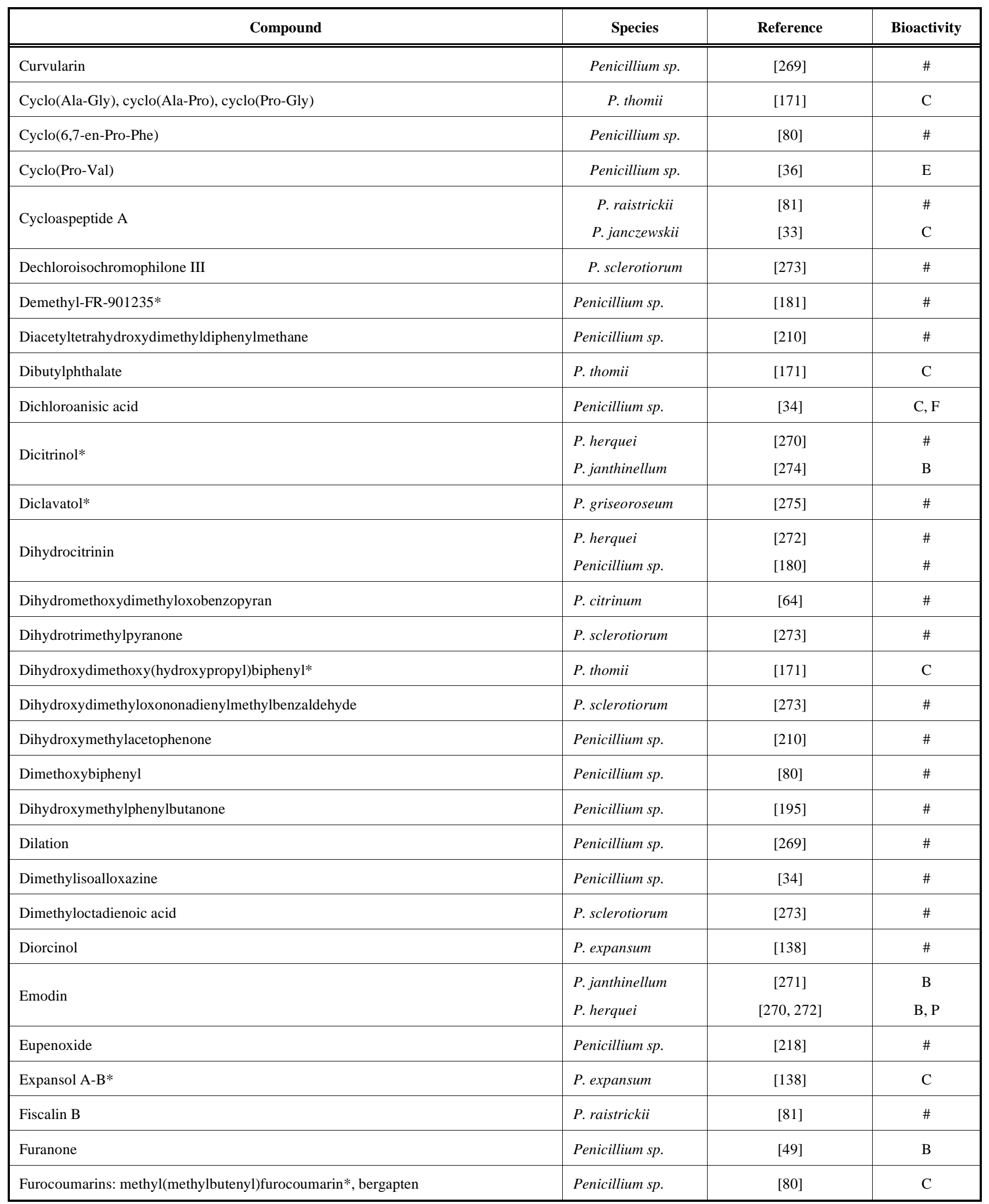


Table 3. contd...

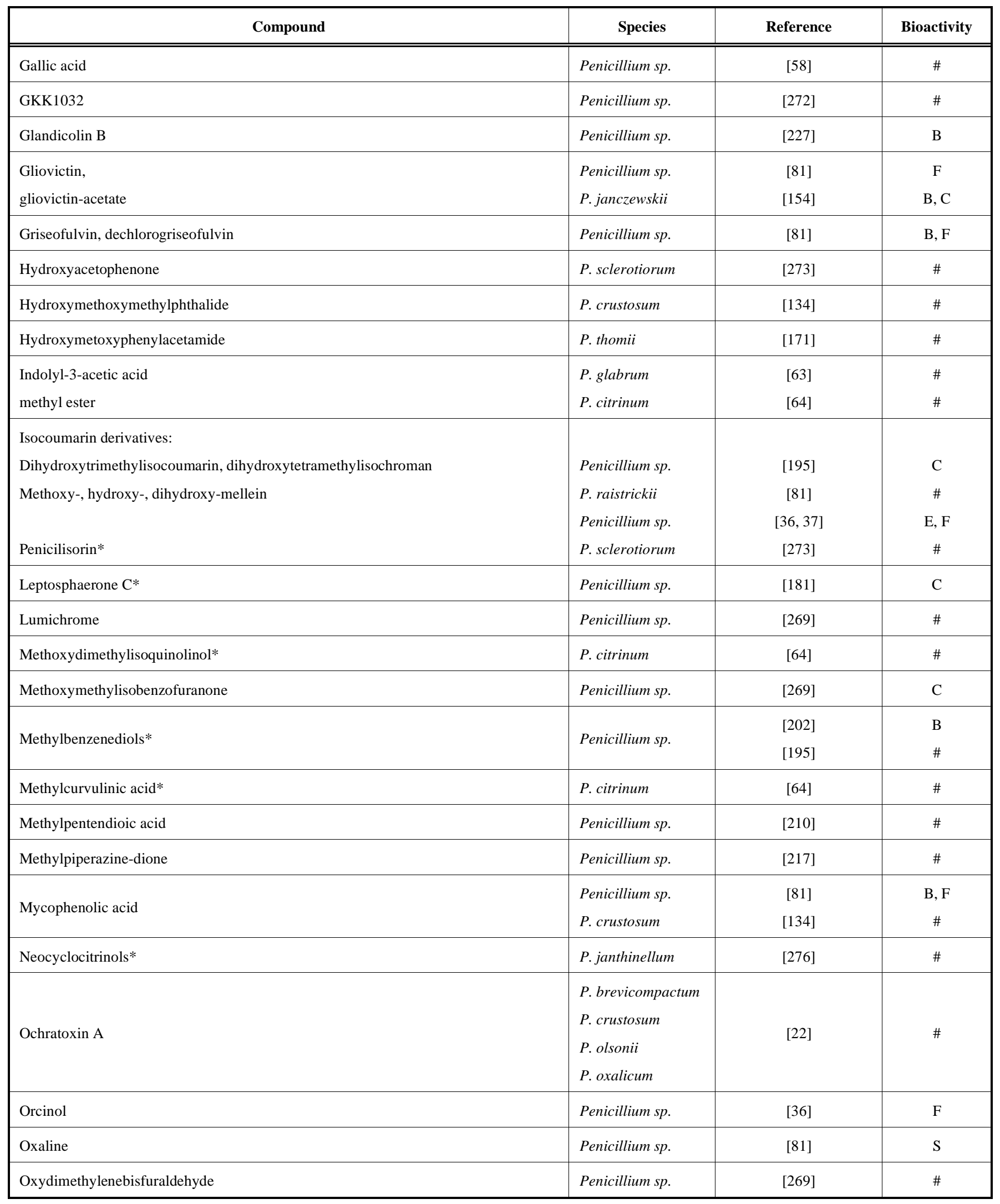


Table 3. contd...

\begin{tabular}{|c|c|c|c|}
\hline Compound & Species & Reference & Bioactivity \\
\hline Paspaline & Penicillium sp. ${ }^{1}$ & [179] & $\#$ \\
\hline Paspalitrem A & Penicillium sp. ${ }^{1}$ & [179] & $\#$ \\
\hline Patulin & Penicillium sp. & {$[81]$} & $\mathrm{B}, \mathrm{F}, \mathrm{S}$ \\
\hline Paxilline & P. paxilli & [277] & $\#$ \\
\hline Pebrolides & Penicillium sp. & {$[81]$} & $\#$ \\
\hline Penicilazaphilone A-B* & P. sclerotiorum & [273] & $\#$ \\
\hline Penicillenols* & Penicillium sp. & {$[180]$} & $\mathrm{C}$ \\
\hline Penicillenone* & Penicillium sp. & [181] & $\mathrm{C}$ \\
\hline $\begin{array}{l}\text { Penicillides: hydroxydidehydropenicillide*, methyldehydroiso-penicillide*, } \\
\text { dehydroisopenicillide, epoxydidehydropenicillide* }\end{array}$ & Penicillium sp. & {$[258]$} & $\mathrm{C}$ \\
\hline Penicillone* & P. paxilli & [277] & $\mathrm{F}$ \\
\hline Penicinoline*, & Penicillium sp. & {$[57]$} & $\mathrm{C}, \mathrm{I}$ \\
\hline Methylpenicinoline & P. citrinum & {$[64]$} & $\#$ \\
\hline Peniprequinolone & P. janczewskii & {$[154]$} & $\mathrm{B}, \mathrm{C}$ \\
\hline Penitrem A-B & P. raistrickii & [81] & $\#$ \\
\hline Phenol A & Penicillium sp. & {$[180,195]$} & $\mathrm{C}$ \\
\hline Phomopsolide A-B, dihydrophomopsolide A-B* & Penicillium sp. & [49] & B \\
\hline Phomoxin B-C* & Penicillium sp. & [218] & \# \\
\hline Physcion & Penicillium sp. & [34] & $\mathrm{F}$ \\
\hline Pseurotin A & $\begin{array}{l}\text { P. raistrickii } \\
\text { P. janczewskii }\end{array}$ & $\begin{array}{l}{[81]} \\
{[33]}\end{array}$ & $\begin{array}{c}\# \\
\mathrm{~B}, \mathrm{C}\end{array}$ \\
\hline Pyrenocine A-B & P. paxilli & [277] & $\mathrm{F}$ \\
\hline Questinol & P. glabrum & [63] & \# \\
\hline Quinolactacide, tetrahydroquinolactacide* & P. citrinum & [64] & \# \\
\hline Quinolins: hydroxyquinolinone, methoxydimethylisoquinolinol* & P. citrinum & [64] & $\#$ \\
\hline Rhodostegone* & P. polonicum & [164] & $\#$ \\
\hline Roquefortine $\mathrm{C}$ & Penicillium sp. & {$[81]$} & \# \\
\hline Rotenone & Penicillium sp. & [55] & I \\
\hline Saponins & Penicillium sp. & [135] & B \\
\hline Sclerotiorin & P. sclerotiorum & {$[273]$} & $\mathrm{F}, \mathrm{V}$ \\
\hline Scopoletin & Penicillium sp. & {$[80]$} & $\#$ \\
\hline Sequoiamonascin D & Penicillium sp. & {$[181]$} & $\#$ \\
\hline Sequoiatone A-B & Penicillium sp. & {$[181]$} & $\#$ \\
\hline Shearinine A, D-K* & Penicillium sp. ${ }^{1}$ & [179] & $\mathrm{K}$ \\
\hline
\end{tabular}


Table 3. contd...

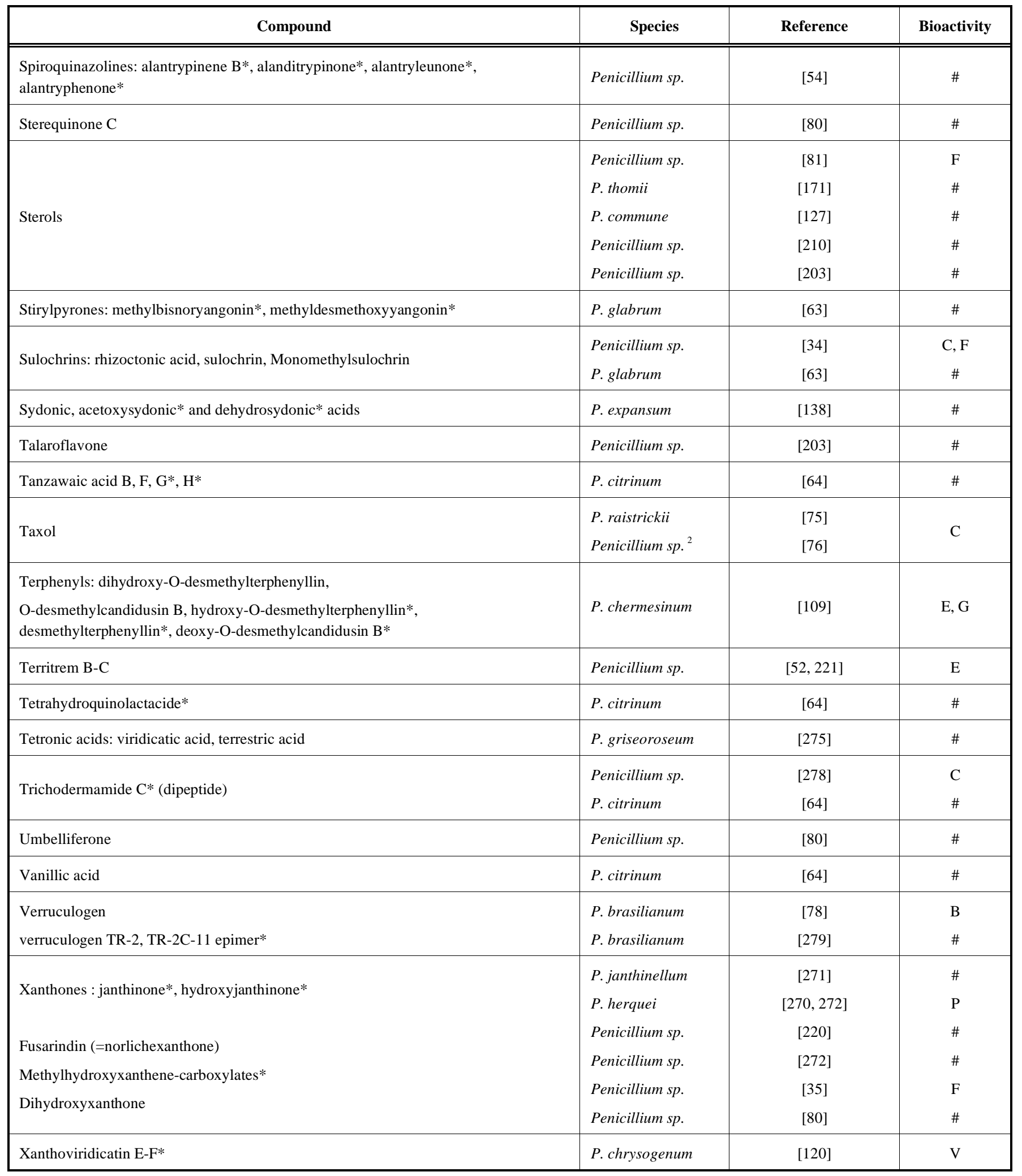

*Compounds identified for the first time in these strains; ${ }^{1}$ closely related to $P$. janthinellum and $P$. simplicissimum; ${ }^{2}$ closely related to $P$. canescens; $\mathrm{B}=$ antibacterial; $\mathrm{C}=$ cytostatic/antiproliferative on tumor cells; $\mathrm{E}=$ acetylcholinesterase inhibitor; $\mathrm{F}=$ antifungal; $\mathrm{G}=\alpha$-glucosidase inhibitor; $\mathrm{I}=\mathrm{insecticidal}$; $\mathrm{K}=\mathrm{blocking}$ calcium-activated potassium channels; $\mathrm{P}=$ antiprotozoal; $\mathrm{S}=$ brine shrimp toxic; $\mathrm{V}=$ antiviral; \#=no activity evaluated in the pertinent reference.

woody plants are given by the efficacy of $P$. glabrum (=P. frequentans) against twig blight of peach trees incited by Monilinia laxa [31], and of $P$. citrinum and $P$. expansum against anthracnose of mango caused by Colletotrichum gloeosporioides [32]. Moreover, some endophytic strains have been characterized for their ability to produce antibiotic 
compounds (Table 3), whose bioactivity against plant pathogens and pests has been documented in a number of cases. Examples are given by pseurotin A produced by $P$. janczewskii against the bacterial pathogens Erwinia carotovora and Pseudomonas syringae [33], and a couple of compounds produced by unidentified Penicillium strains, such as dichloroanisic acid against Aspergillus niger [34], and dimethyl-8-methoxy-9-oxo-9H-xanthene-1,6-dicarboxylate against Fusarium oxysporum f. sp. cubense [35]. More products from unidentified strains possibly involved in the defense of their host species against infection by pathogenic fungi are orcinol and 4-hydroxymellein [36], and a few dihydroisocoumarins [37]. Otherwise, the production of antifungal extrolites may be presumed based on inhibitory properties in dual cultures, which is a condition reported for a number of strains/species such as $P$. citrinum, $P$. multicolor and $P$. oxalicum isolated from Abies beshanzuensis [38], $P$. adametzi and $P$. chrysogenum from Justicia wayanadensis [39], $P$. chrysogenum and P. corylophilum from Calotropis procera [40], P. commune from olive tree (Olea europaea) [41], and $P$. aurantiogriseum, $P$. commune, P. echinulatum, $P$. expansum and $P$. viridicatum from cupressaceous hosts [42].

On the insect pest side, a role in protection of white spruce (Picea glauca) against the spruce budworm (Choristoneura fumiferana) has been given to endophytic fungi producing rugulosin [43], an anthraquinone compound which is also known as a secondary metabolite of a few Penicillium species [44]. More in general, anthraquinones are believed to contribute to the defense of plants towards pests and disease agents [45], and besides a known occurrence as plant metabolites, additional compounds of this class such as citrorosein, emodin, and physcion have been reported by several endophytic Penicillium strains (Table 3). The same consideration pertains the structurally related dibenzo- $\gamma$-pyrones, or xanthones (Table $\mathbf{3}$ ), which are known for their insecticidal effect and have been reported from plant species belonging to at least 20 botanical families [46]. The azadirachtins, tetranortriterpene limonoids typical of the neem tree (Azadirachta indica) and related species in the Meliaceae, represent a well known example of natural insecticide mainly acting as an antifeedant and a growth disruptor, which have been recently found to be produced by an endophytic strain of $P$. (Eupenicillium) parvum [47]. Likewise, antifeedant effects against the elm bark beetles (Scolyuts spp.) have been reported for the phomopsolides, pyranone compounds first characterized from Phomopsis oblonga [48], and later extracted from liquid cultures of an unidentified endophytic Penicillium strain [49]. Insecticidal properties have been also pointed out for the brasiliamides by an endophytic strain of $P$. brasilianum, which were found to induce convulsive effects in silkworms (Bombyx mori) [50] similar to those referable to penitrems [51] and other tremorgenic meroterpenoids of Penicillium. The latter class of compounds also includes the arisugacins and the territrems recently reported by a strain from Tamarix chinensis [52]. Alanditrypinone and related spiroquinazoline alkaloids, well known for their insecticidal activity [53], have been found as secondary metabolites of a strain (Eupenicillium sp.) recovered from leaves of Murraya paniculata [54]. Moreover, strains (Penicillium sp.) recovered from root of Derris elliptica [55], and from leaf of Derris hancei [56], have been reported to produce metabolites displaying insecticidal activity against larvae of noxious moths (Plutella xylostella and Spodoptera litura), and against the turnip aphid (Lipaphis erysimi). Rotenone, or a related product, was identified among these compounds [55]. Finally, insecticidal activity against the melon and cotton aphid (Aphis gossypii) has been reported for penicinoline, a novel pyrrolyl 4-quinolinone alkaloid produced by another unidentified strain [57].

Besides a direct antibiotic role, some endophyte metabolites are also considered to possibly act as elicitors inducing enhanced plant protection against biotic adversities. Production of gallic acid by an endophytic Penicillium strain has been reported with reference to such a positive effect [58]. Other endophytes may influence plant development in consequence of their ability to release phytohormones, among which gibberellins, primarily known as fungal metabolites, are particularly considered for their implication in overcoming the adverse effects of abiotic stresses [59]. Within Penicillium, production of gibberellins has been observed by endophytic strains from herbaceous plants [6062], while so far there is no direct evidence for strains recovered from trees and shrubs. Conversely, production of auxins has been reported by endophytic strains of $P$. glabrum [63] and $P$. citrinum [64], respectively from pomegranate (Punica granatum) and carob tree (Ceratonia siliqua).

Mutualism may also involve at least part of the multitude of Penicillium strains/species inhabiting rhizosphere which are supposed to be eventually involved in mycorrhizal relationships on tree roots. Actually, this symbiotic context is mostly conjectural, considering that a review of the huge literature available on the subject [65] refers an unproved establishment of Penicillium strains in the root tissues. A notable exception is represented by the particular interaction occurring on alder roots, consisting in the induction of the formation of myconodules confined at the outer cortical layer. A fungus inducing myconodules on Alnus incana was first identified as $P$. albidum [66]. Afterwards, isolates recovered from roots of Alnus glutinosa were ascribed to the closely related $P$. nigricans [67], currently treated as a synonym of $P$. janczewskii. The identity of alder isolates as a new species was later advanced [68], and the name Penicillium nodositatum proposed [69]. It was found to be phylogenetically related to species in the subgenus Biverticillium [70], but not included in the mentioned taxonomic revision by Samson, et al. [8]. Considering the unknown functions of myconodules and the absence of any apparent damage to alder plants despite the observed death of cortical cells following fungal infection, $P$. nodositatum has been defined as a neutral microsymbiont [71]. Other observations have pointed out that it may rather act as a competitor at the root infection sites against actinorhizae promoted by Frankia strains establishing a mutualistic interaction based on nitrogen fixation [68, 72]. The association with alder roots resulting in samplings from several European countries (Table 1) was indicative of an occurrence of this species confined to Alnus spp.; however, this hypothesis is destined to be abandoned in consequence of a more recent finding as an endophyte in leaves of twinflower (Linnea borealis) [73]. 


\section{Neutral Interactions}

The biocenotic interaction involving endophytes that do not apparently exert any particular effect on host plants can be defined as neutral, or based on a commensalitic aptitude. Awaiting further investigations which may eventually disclose unknown aspects concerning their effective role, this condition is inherent to the majority of the endophytic Penicillium strains recovered so far.

The available data aggregated for species (Table 1) are not indicative of a host specialization or preference. Adaptation to a particular plant species might be conceived at some extent for strains that have resulted to produce secondary metabolites originally characterized from their host, in a relationship which possibly reflects a horizontal gene transfer between the two bionts [74]. Within a recently consolidated research trend aimed at identifying fungal endophytes expressing this particular aptitude to be exploited as an alternative and sustainable source, a number of Penicillium strains have been reported for the production of important plant-derived drugs. In fact, this is the case of the taxol-producing strains of $P$. raistrickii from the Pacific yew (Taxus brevifolia) [75], and Penicillium sp. from the Mexican yew (Taxus globosa) [76], of two unidentified isolates from Cinchona ledgeriana producing cinchonin and related alkaloids [77], and of the above-mentioned strain of $P$. parvum from $A$. indica producing azadirachtins [47]. Moreover, an endophytic Penicillium sp. from another azadirachtin source, the chinaberry tree (Melia azedarach), has been reported to produce the structurally related austins [78], indicating their possible derivation from a common biosynthetic pathway. It is also interesting to mention the finding of an endophytic strain of $P$. griseoroseum from coffee seeds able to synthesize a benzylated flavanoid when the compound pentamethoxyflavanone was added to the growth medium [79], which demonstrates that endophytes may exert their biosynthetic potential by modifying plant metabolites to generate products that are not ordinarily produced by the plants themselves.

In the field of biosynthetic affinities, there are also examples of known plant metabolites reported from endophytes of unrelated plants. This is the case of bergapten, occurring in bergamot essential oil and in grapefruit juice, that has been recently found as a product of a mangrove endophytic Penicillium strain [80]. Likewise asperphenamate, an anticancer compound reported from plants belonging to several unrelated families, is produced by the mentioned industrious and versatile strain H10BA2 of $P$. raistrickii from $T$. brevifolia [81], and is part of the pattern of extrolites characterizing Penicillium species in the section Brevicompacta [82], some of which are also known with reference to a facultative endophytic occurrence.

References for 66 Penicillium species and many unidentified strains recovered as endophytes are listed in Tables 1 and 2. Within the identified strains, not surprisingly a higher number of records pertain to ubiquitous species, such as $P$. brevicompactum, $P$. chrysogenum, $P$. citrinum and $P$. glabrum, which confirm their ability to virtually colonize any kind of environment on earth. However, species identification has not been accomplished in as much as $43 \%$ of the findings, reflecting both the difficulty to get to a reliable ascription through the conventional methods, and the likely existence of novel species awaiting to be classified. According to the new taxonomic scheme, a good proportion of these isolates could actually belong to Talaromyces. Unfortunately, this possibility cannot be further investigated for the majority of such material, which introduces the opportunity to define appropriate description standards to be followed in reports concerning novel isolates of problematic taxonomic placement. Undoubtedly, more detailed investigations on the role and properties of endophytic strains cannot overlook a thorough taxonomic referencing.

On the plant side, a total number of 195 species are reported as hosts of endophytic Penicillium strains. Most of these trees and shrubs harbor just one or two species, but it is evident that more systematic investigations might disclose more composed assemblages, as happened for coffee. Occasionally the plant source has not been specified, such as in a few papers considering groups of medicinal and mangrove plants whose authors missed to relate the isolated strains to the pertinent botanical species [83-87]. Medicinal plants are more and more considered in view of the possible involvement of fungal endophytes in the expression of their favourable pharmacological effects [88]. Indeed, the search for novel pharmaceuticals has stimulated a huge investigational activity aiming at the exploitation of microbial sources, the reason why an increasing number of reports come from tropical habitats such as the mangrove forests [89, 90]. As inferable by a number of pertinent citations, the occurrence of endophytic manglicolous fungi has been intensively investigated in China and South-east Asia; unfortunately, additional data published on local journals could not be accessed, hence not considered in this manuscript. The context of temperate forests has been also diffusely examined in a number of studies, disclosing a few interesting cases of mutualistic interactions as mentioned above [91]. Conversely, reports concerning crop plants are quite fragmentary. Apart, the already mentioned exception of coffee, and a couple of plantation species such as cocoa and rubber tree (Hevea brasiliensis), the few occasional citations from grapevine (Vitis vinifera), kiwifruit (Actinidia chinensis), olive and pomegranate (Tables $\mathbf{1}$ and 2) are indicative of an underestimation of the role of endophytes in crops, which is awaiting to be reconsidered particularly in view of a more thorough appreciation of the concept of defensive mutalism. Indeed, appropriate investigations on the ability to release bioactive metabolites in vivo could disclose interesting effects on the host plant, and foster additional applications of Penicillium strains in plant protection.

\section{APPLICATIVE ISSUES OF BIOACTIVE SECON- DARY METABOLITES}

Directly or indirectly, a substantial part of the work carried out worldwide concerning endophytism in Penicillium aimed at the characterization of secondary metabolites, and their bioactivity. This has resulted in a huge 
<smiles>C[C@H]1Cc2c(Cl)cc(C(=O)N[C@@H](Cc3ccccc3)C(=O)O)c(O)c2C(=O)O1</smiles>

ochratoxin A

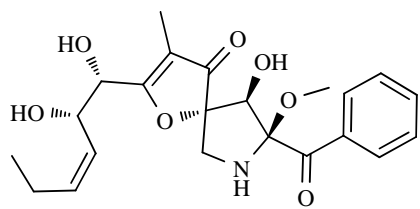

pseurotin A<smiles>COc1c(Cl)cc(C(=O)O)cc1Cl</smiles>

dichloroanisic acid<smiles>Cc1cc(O)cc(O)c1</smiles>

orcinol<smiles>COC(=O)c1cc(OC)c2c(=O)c3c(C(=O)OC)cccc3oc2c1</smiles>

dimethyl-8-methoxy-9-oxo-9Hxanthene-1,6-dicarboxylate

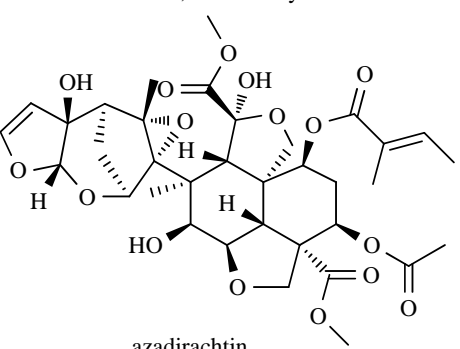<smiles>COc1ccc(-c2cc3c(c(=O)o2)C[C@]2(C)[C@]4(O)C(=O)C=CC(C)(C)C4CC[C@@]2(C)O3)cc1OC</smiles>

arisugacin A<smiles>C[C@@H]1OC(=O)c2c(O)cccc2[C@H]1O</smiles>

4-hydroxymellein<smiles>Cc1cc(O)c2c(c1)C(=O)C13C(=O)c4cc(C)cc(O)c4C4(C(O)=C(O)C(O)C1C4(O)O)C(O)C(O)=C3C2=O</smiles>

rugulosin<smiles>C/C=C(\C)C(=O)O[C@H]1C=CC(=O)O[C@H]1/C=C/[C@H](O)[C@H](C)O</smiles><smiles>O=C(O)c1cc(O)c(O)c(O)c1</smiles>

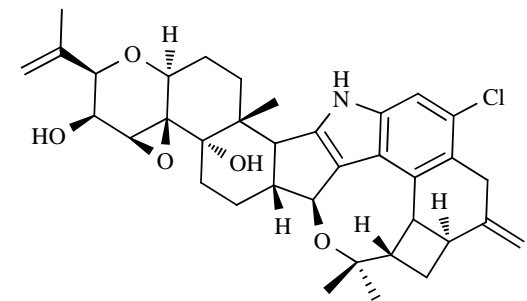<smiles></smiles>

territrem B

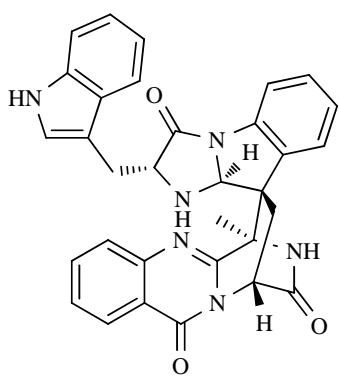<smiles>O=C(O)c1c(-c2ccc[nH]2)[nH]c2ccccc2c1=O</smiles>

penicinoline

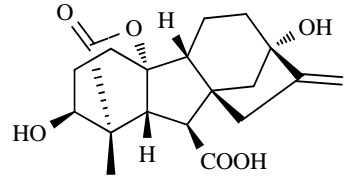

gibberellin A1

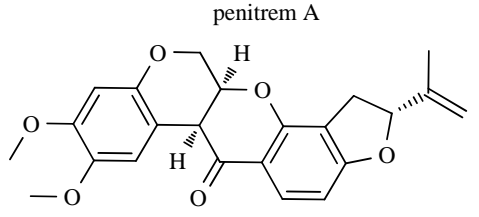

$\begin{array}{ll}\text { emodin } & \mathrm{R}=\mathrm{H} \\ \text { physcion } & \mathrm{R}=\mathrm{Me}\end{array}$<smiles>[R2]c1cc(O)c2c(c1)C(=O)c1cc(C)cc(O)c1C2=O</smiles>

. 
etc. [96, 97]. However, a grouping based on their functional and bioactive properties can be more useful for an applicative viewpoint. An alphabetic list is provided in Table 3, including a relevant proportion of compounds, approximately $33 \%$, first discovered from these strains. Their bioactivity has been assayed in multiple fields, disclosing antibiotic, cytostatic, insecticidal, and other miscellaneous properties, which of course are not intended to have been systematically investigated for each molecule. Thus, additional data are expected to be progressively integrated in view of a more thorough characterization of their biological effects. To this regard, readers should consider that this review covers endophytic Penicillium strains only; therefore, data summarized in Table 3 do not account for bioactivities resulting when these compounds were eventually extracted from other fungal strains or producing organisms.

\section{CONCLUSION}

The many and varied issues introduced with this overview announce future developments in studies concerning endophytic Penicillium strains. Indeed, quite intriguing aspects are involved covering diverse but interrelated disciplines, such as microbial ecology, plant pathology, biochemistry, food processing and pharmacology. A thorough knowledge of the state of the art is fundamental in order to enhance qualitative and quantitative detection of these fungi within endophytic communities, and to improve our ability to elucidate their ecological functions. Thus, data collected so far represent a valuable heritage to draw upon, both for the general purpose to expand the available information concerning Penicillium species, and for the more specific objective of exploiting these strains as a true microbial factory, yielding a largely untapped reservoir of chemically diverse natural products which have been optimized as effective bioactive agents by the pressure of evolutionary and environmental factors.

\section{CONFLICT OF INTEREST}

The authors confirm that this article content has no conflict of interest.

\section{ACKNOWLEDGEMENTS}

Declared none.

\section{REFERENCES}

[1] Hirsch G, Braun U. Communities of parasitic microfungi. In: Winterhoff W, Ed. Handbook of vegetation science. Vol. 19. Fungi in vegetation science. Dordrecht, Kluwer Academic Publishers 1992; pp. 225-50.

[2] Whitham TG, Young WP, Martinsen GD, et al. Community and ecosystem genetics: a consequence of the extended phenotype. Ecology 2003; 84: 559-73.

[3] Yuan ZL, Zhang CL, Lin FC. Role of diverse non-systemic fungal endophytes in plant performance and response to stress: progress and approaches. J Plant Growth Regul 2010; 29: 116-26.

[4] Schulz B, Boyle C. The endophytic continuum. Mycol Res 2005; 109: 661-86.
[5] Ganley RJ, Brunsfeld SJ, Newcombe G. A community of unknown, endophytic fungi in western white pine. Proc Nat Acad Sci USA 2004; 101: 10107-12.

[6] Gunatilaka AAL. Natural products from plant-associated microorganisms: distribution, structural diversity, bioactivity, and implications of their occurrence. J Nat Prod 2006; 69: 509-26.

[7] Houbraken J, Samson RA. Phylogeny of Penicillium and the segregation of Trichocomaceae into three families. Stud Mycol 2011; 70: 1-51.

[8] Samson RA, Yilmaz N, Houbraken J, et al. Phylogeny and nomenclature of the genus Talaromyces and taxa accommodated in Penicillium subgenus Biverticillium. Stud Mycol 2011; 70: 159-83.

[9] Ge HM, Shen Y, Zhu CH, et al. Penicidones A-C, three cytotoxic alkaloidal metabolites of an endophytic Penicillium sp. Phytochemistry 2008; 69: 571-6.

[10] Kubatova A. Neglected Penicillium spp. associated with declining trees. In: Samson RA, Pitt JI, Eds. Integration of modern taxonomic methods for Penicillium and Aspergillus classification. Amsterdam, Harwood Academic Publishers 2004; pp. 299-307.

[11] Summerbell RC. Root endophyte and mycorrhizosphere fungi of black spruce, Picea mariana, in a boreal forest habitat: influence of site factors on fungal distributions. Stud Mycol 2005; 53: 121-45.

[12] Rodriguez RJ, White JF Jr, Arnold AE, Redman RS. Fungal endophytes: diversity and functional roles. New Phytol 2009; 182: 314-30.

[13] Holmes GJ, Eckert JW, Pitt JI. Revised description of Penicillium ulaiense and its role as a pathogen of citrus fruits. Phytopathology 1994; 84: 719-27.

[14] Sanderson PG, Spotts RA. Post-harvest decay of winter pear and apple fruit caused by species of Penicillium. Phytopathology 1995; 85: $103-10$

[15] Shim JO, Choi KD, Hahn KD, et al. Blue mold of pear caused by Penicillium aurantiogriseum in Korea. Mycobiology 2002; 30: 105-6.

[16] Labuda R, Hudec K, Piecková E, et al. Penicillium implicatum causes a destructive rot of pomegranate fruits. Mycopathologia 2004; 157: 217-23.

[17] Serey RA, Torres R, Latorre BA. Pre- and post-infection activity of new fungicides against Botrytis cinerea and other fungi causing decay of table grapes. Ciencia Investig Agr 2007; 34: 215-24.

[18] Bardas GA, Tzelepis GD, Lotos L, Karaoglanidi GS. First report of Penicillium glabrum causing fruit rot of pomegranate (Manilkara bidentata) in Greece. Plant Dis 2009; 93: 1347.

[19] La Guerche S, Garcia C, Darriet P, Dubourdieu D, Labarère J. Characterization of Penicillium species isolated from grape berries by their internal transcribed spacer (ITS1) sequences and by gas chromatography-mass spectrometry analysis of geosmin production. Curr Microbiol 2004; 48: 405-11.

[20] Paterson RRM, Archer S, Kozakiewicz Z, Lea A, Locke T, O'Grady E. A gene probe for the patulin metabolic pathway with potential for use in patulin and novel disease control. Biocontr Sci Technol 2000; 10: 509-12.

[21] Díaz GA, Yañez L, Latorre BA. Low occurrence of patulinproducing strains of Penicillium in grapes and patulin degradation during wine making in Chile. Am J Enol Viticul 2011; 62: 542-6.

[22] Vega FE, Posada F, Peterson SW, Gianfagna TJ, Chaves F. Penicillium species endophytic in coffee plants and ochratoxin A production. Mycologia 2006; 98: 31-42.

[23] Peterson SW, Vega FE, Posada F, Nagai C. Penicillium coffeae, a new endophytic species isolated from a coffee plant and its phylogenetic relationship to $P$. fellutanum, $P$. thiersii and $P$. brocae based on parsimony analysis of multilocus DNA sequences. Mycologia 2005; 97: 659-66.

[24] CBS strain collection by the Fungal Biodiversity Centre. Utrecht, the Netherlands [cited: $9^{\text {th }}$ Dec 2013]. Available from: http://www.cbs.knaw.nl/Collections/Biolomics.aspx?Table=CBS\% 20strain\%20database

[25] Seifert KA, Hoekstra ES, Frisvad JC, Louis-Seize G. Penicillium cecidicola, a new species on cynipid insect galls on Quercus pacifica in the western United States. Stud Mycol 2004; 50: 51723.

[26] Wilson D. Fungal endophytes which invade insect galls: insect pathogens, benign saprophytes, or fungal inquilines? Oecologia 1995; 103: 255-60.

[27] Joy JB. Symbiosis catalyses niche expansion and diversification. Proc Biol Sci 2013; 280: 20122820; doi: 10.1098/rspb.2012.2820 
[28] White JF, Torresa MS. Is plant endophyte-mediated defensive mutualism the result of oxidative stress protection? Physiol Plantarum 2010; 138: 440-6.

[29] Panaccione DG, Beaulieu WT, Cook D. Bioactive alkaloids in vertically transmitted fungal endophytes. Funct Ecol 2014; 28: 299314.

[30] Hanada RE, Pomella AWV, Costa HS, et al. Endophytic fungal diversity in Theobroma cacao (cacao) and $T$. grandiflorum (cupuaçu) trees and their potential for growth promotion and biocontrol of black-pod disease. Fungal Biol 2010; 114: 901-10.

[31] de Cal A, Sagasta EM, Melgarejo P. Biological control of peach twig blight (Monilinia laxa) with Penicillium frequentans. Plant Pathol 1990; 39: 612-8.

[32] Moromizato Z, Takushi T, Taba S, Adaniya S, Motomura K. The study on the biological control of mango anthracnose: (I) microflora on mango leaves and screening of antagonists. Jpn J Tropic Agr 2003; 47: 34-41.

[33] Schmeda-Hirschmann G, Hormazabal E, Rodriguez JA, Theoduloz C. Cycloaspeptide A and pseurotin A from the endophytic fungus Penicillium janczewskii. Z Naturforsch 2008; 63c: 383-8.

[34] Wang FW, Hou ZM, Wang CR, Li P, Shi DH. Bioactive metabolites from Penicillium sp., an endophytic fungus residing in Hopea hainanensis. World J Microbiol Biotechnol 2008; 24: 2143-7.

[35] Shao C, Wang C, Wie M, et al. Structure elucidation of two new xanthone derivatives from the marine fungus Penicillium sp. (ZZF 32\#) from the South China Sea. Magn Reson Chem 2008; 46: 1066-9.

[36] Oliveira CM, Silva GH, Regasini LO, et al. Bioactive metabolites produced by Penicillium sp. 1 and sp. 2, two endophytes associated with Alibertia macrophylla (Rubiaceae). Z Naturforsch 2009; 64c: 824-30.

[37] Oliveira CM, Regasini LO, Silva GH, et al. Dihydroisocoumarins produced by Xylaria sp. and Penicillium sp., endophytic fungi associated with Alibertia macrophylla and Piper aduncum. Phytochem Lett 2011; 4: 93-6.

[38] Yuan ZL, Rao LB, Chen YC, Zhang CL, Wu YG. From pattern to process: species and functional diversity in fungal endophytes of Abies beshanzuensis. Fungal Biol 2011; 115: 197-213.

[39] Vinu AK, Jayashankara M. Potentiality of endophytic fungi of Justicia wayanadensis as bioagent against Rhizoctonia solani. Asian J Microbiol Biotechnol Environ Sci 2011; 13: 7-9.

[40] Gherbawy YA, Gashgari RM. Molecular characterization of fungal endophytes from Calotropis procera plants in Taif region (Saudi Arabia) and their antifungal activities. Plant Biosyst 2013; doi: 10.1080/11263504.2013.819043

[41] Martins F, Pereira JA, Bento A, Baptista P. Potentialities of endophytic fungi of olive tree as biological control agents against Colletotrichum acutatum and Verticillium dahliae. In: Schneider C, Leifert C, Feldmann F, Eds. Endophytes for plant protection: the state of the art. Braunschweig, Deutsche Phytomedizinische Gesellschaft 2013; p. 190

[42] Moghaddam MSH, Soltani J, Babalhavaeji F, Hamzei J, Nazeri S, Mirzaei S. Bioactivities of endophytic Penicillia from Cupressaceae. J Crop Prot 2013; 2: 421-33.

[43] Miller JD, Mackenzie S, Foto M, Adams GW, Findlay JA. Needles of white spruce inoculated with rugulosin-producing endophytes contain rugulosin reducing spruce budworm growth rate. Mycol Res 2002; 106: 471-9.

[44] Nicoletti R, Ciavatta ML, Buommino E, Tufano MA. Antitumor extrolites produced by Penicillium species. Int J Biomed Pharm Sci 2008; 2: 1-23.

[45] Gessler NN, Egorova AS, Belozerskaya TA. Fungal anthraquinones. Appl Biochem Microbiol 2013; 49: 85-99.

[46] Vieira LMM, Kijjoa A. Naturally-occurring xanthones: recent developments. Curr Med Chem 2005; 12: 2413-46.

[47] Kusari S, Verma VC, Lamshoeft M, Spiteller M. An endophytic fungus from Azadirachta indica A. Juss. that produces azadirachtin. World J Microbiol Biotechnol 2012; 28: 1287-94.

[48] Grove JF. Metabolic products of Phomopsis oblonga. Part 2. Phomopsolide A and B, tiglic esters of two 6-substituted 5,6dihydro-5-hydroxypyran-2-ones. J Chem Soc Perkin Trans 1985; 1 : 865-9.

[49] Stierle DB, Stierle AA, Ganser B. New phomopsolides from a Penicillium sp. J Nat Prod 1997; 60: 1207-9.
[50] Fujita T, Makishima D, Akiyama K, Hayashi H. New convulsive compounds, brasiliamides $\mathrm{A}$ and $\mathrm{B}$, from Penicillium brasilianum Batista JV-379. Biosci Biotechnol Biochem 2002; 66: 1697-705.

[51] González MC, Lull C, Moya P, Ayala I, Primo J, Primo YE. Insecticidal activity of penitrems, including penitrem $\mathrm{G}$, a new member of the family isolated from Penicillium crustosum. J Agr Food Chem 2003; 51: 2156-60.

[52] Sun X, Kong X, Gao H, et al. Two new meroterpenoids produced by the endophytic fungus Penicillium sp. SXH-65. Arch Pharm Res 2013; doi: 10.1007/s12272-013-0268-2

[53] Watanabe T, Arisawa M, Narusuye K, et al. Alantrypinone and its derivatives: synthesis and antagonist activity toward insect GABA receptors. Bioorg Med Chem 2009; 17: 94-110.

[54] Proença Barros FA, Rodrigues-Filho E. Four spiroquinazoline alkaloids from Eupenicillium sp. isolated as an endophytic fungus from leaves of Murraya paniculata (Rutaceae). Biochem Syst Ecol 2005; 33: 257-68.

[55] Hu MY, Zhong GH, Sun ZT, Sh G, Liu HM, Liu XQ. Insecticidal activities of secondary metabolites of endophytic Penicillium in Derris elliptica Benth. J Appl Entomol 2005; 129: 413-7.

[56] Huang XP, Luo JJ, Hu MY, Liang YY, Ye XX, Zhong GH. Isolation and bioactivity of endophytic fungi in Derris hancei. J South China Agr Univ 2009; 30: 44-7.

[57] Shao CL, Wang CY, Gu YC, et al. Penicinoline, a new pyrrolyl 4quinolinone alkaloid with an unprecedented ring system from an endophytic fungus Penicillium sp. Bioorg Med Chem Lett 2010; 20: 3284-6.

[58] Qi FH, Jing TZ, Wang ZX, Zhan YG. Fungal endophytes from Acer ginnala Maxim: isolation, identification and their yield of gallic acid. Lett Appl Microbiol 2009; 49: 98-104.

[59] Khan AL, Hussain J, Al-Harrasi A, Al-Rawahi A, Lee IJ. Endophytic fungi: resource for gibberellins and crop abiotic stress resistance. Crit Rev Biotechnol 2013; doi:10.3109/07388551.2013.800018

[60] Khan SA, Hamayun M, Yoon H, et al. Plant growth promotion and Penicillium citrinum. BMC Microbiol 2008; 8: 231.

[61] Hamayun M, Khan SA, Iqbal I, Ahmad B, Lee IJ. Isolation of a gibberellin-producing fungus (Penicillium sp. MH7) and growth promotion of crown daisy (Chrysanthemum coronarium). J Microbiol Biotechnol 2010; 20: 202-7.

[62] Waqas M, Khan AL, Kamran M, et al. Endophytic fungi produce gibberellins and indoleacetic acid and promotes host-plant growth during stress. Molecules 2012; 17: 10754-73.

[63] Hammerschmidt L, Wray V, Lin W, Kamilova E, Proksch P, Aly AH. New styrylpyrones from the fungal endophyte Penicillium glabrum isolated from Manilkara bidentata. Phytochem Lett 2012; 5: 600-3.

[64] El-Neketi M, Ebrahim W, Lin W, et al. Alkaloids and polyketides from Penicillium citrinum, an endophyte isolated from the Moroccan plant Ceratonia siliqua. J Nat Prod 2013; 76: 1099-104.

[65] Summerbell RC. From Lamarckian fertilizers to fungal castles: recapturing the pre-1985 literature on endophytic and saprotrophic fungi associated with ectomycorrhizal root systems. Stud Mycol 2005; 53: 191-256.

[66] Pommer EH. Beiträge zur anatomie und biologie der wurzelknöllchen von Alnus glutinosa Gaertn. Flora (Jena) 1956; 143: 604-34.

[67] Van Dijk C. Ecological aspects of spore formation in the FrankiaAlnus symbiosis. Ph.D. Thesis, University of Leiden 1984; p. 154.

[68] Capellano A, Dequatre B, Valla G, Moiroud A. Root-nodules formation by Penicillium sp. on Alnus glutinosa and Alnus incana. Plant Soil 1987; 104: 45-51.

[69] Valla G, Capellano A, Hugueney R, Moiroud A. Penicillium nodositatum Valla, a new species inducing myconodules on Alnus roots. Plant Soil 1989; 114: 142-6.

[70] Sequerra J, Marmeisse R, Valla G, Normand P, Capellano A, Moiroud A. Taxonomic position and intraspecific variability of the nodule forming Penicillium nodositatum inferred from RFLP analysis of the ribosomal intergenic spacer and RAPD. Mycol Res 1997; 101: 465-72.

[71] Sequerra J, Capellano A, Gianinazzi-Pearson V, Moiroud A. Ultrastructure of cortical root cells of Alnus incana infected by Penicillium nodositatum. New Phytol 1995; 130, 545-55.

[72] Wolters DJ, van Dijk C, Akkermans ADL, Woldendorp JW. Ineffective Frankia and host resistance in natural populations of Alnus glutinosa (L.) Gaertn. Acta Oecol 1999; 20: 71-9. 
[73] Stone JK, Polishook JD, White JF. Endophytic fungi. In: Foster M, Bills G, Eds. Biodiversity of fungi. Burlington, Elsevier Academic Press 2004; pp. 241-70.

[74] Gutierrez RMP, Gonzalez AMN, Ramirez AM. Compounds derived from endophytes: a review of phytochemistry and pharmacology. Curr Med Chem 2012; 19: 2992-3030.

[75] Stierle AA, Stierle DB, Strobel G, Bignami G, Grothaus P. Bioactive metabolites of the endophytic fungi of Pacific yew, Taxus brevifolia: paclitaxel, taxanes, and other bioactive compounds. In: Georg GI, Chen TT, Ojima I, Vyas DM, Eds. Taxane anticancer agents: basic science and current status. Washington, American Chemical Society 1995; pp. 81-97.

[76] Soca-Chafre G, Rivera-Orduña FN, Hidalgo-Lara ME, HernandezRodriguez C, Marscha R, Flores-Cotera LB. Molecular phylogeny and paclitaxel screening of fungal endophytes from Taxus globosa. Fungal Biol 2011; 115: 153-6.

[77] Maehara S, Simanjuntak P, Maetani Y, Kitamura C, Ohashi K, Shibuya H. Ability of endophytic filamentous fungi associated with Cinchona ledgeriana to produce Cinchona alkaloids. J Nat Med 2013; 67: 421-3.

[78] Geris dos Santos RM, Rodrigues-Fo E. Meroterpenes from Penicillium sp. found in association with Melia azedarach. Phytochemistry 2002; 61: 907-12.

[79] Ferreira da Silva B, Rodrigues-Fo E. Production of a benzylated flavonoid from 5,7,3',4',5'-pentamethoxyflavanone by Penicillium griseoroseum. J Mol Catal B: Enzym 2010; 67: 184-8.

[80] Huang Z, Yang J, Cai X, She Z, Lin Y. A new furanocoumarin from the mangrove endophytic fungus Penicillium sp. (ZH16). Nat Prod Res 2012; 26: 1291-5.

[81] Stierle AA, Stierle DB. Bioactive compounds from four endophytic Penicillium sp. of a Northwest Pacific yew tree. Stud Nat Prod Chem 2000; 24: 933-77.

[82] Frisvad JC, Houbraken J, Popma S, Samson RA. Two new Penicillium species Penicillium buchwaldii and Penicillium spathulatum, producing the anticancer compound asperphenamate. FEMS Microbiol Lett 2013; 339: 77-92.

[83] Shankar Naik B, Shashikala J, Krishnamurthy YL. Diversity of fungal endophytes in shrubby medicinal plants of Malnad region, Western Ghats, Southern India. Fungal Ecol 2008; 1: 89-93.

[84] Theantana T, Hyde KD, Lumyong S. Asparaginase production by endophytic fungi from Thai medicinal plants: cytotoxic properties. Int J Integr Biol 2009; 7: 1-8.

[85] Bharathidasan R, Panneerselvam A. Biosynthesis and characterization of silver nanoparticles using endophytic fungi Aspergillus concius, Penicillium janthinellum and Phomosis sp. Int J Pharm Sci Res 2012; 3: 3163-9.

[86] Anitha D, Vijaya T, Pragathi D, et al. Isolation and characterization of endophytic fungi from endemic medicinal plants of Tirumala hills. Int J Life Sci Biotechnol Pharma Res 2013; 2: 367-73.

[87] de Souza SFL, Romão-Dumaresq AS, Lacava PT, et al. Species diversity of culturable endophytic fungi from Brazilian mangrove forests. Curr Gen 2013; 59: 153-66.

[88] Chowdhary K, Kaushik N, Coloma AG, Raimundo CM. Endophytic fungi and their metabolites isolated from Indian medicinal plant. Phytochem Rev 2012; 11: 467-85.

[89] Debbab A, Aly AH, Proksch P. Mangrove derived fungal endophytes-a chemical and biological perception. Fungal Diver 2013; 61: 1-27.

[90] Thatoi H, Behera BC, Mishra RR. Ecological role and biotechnological potential of mangrove fungi: a review. Mycology 2013; 4: 54-71.

[91] Sieber TN. Endophytic fungi in forest trees: are they mutualists? Fungal Biol Rev 2007; 21: 75-89.

[92] Gosio B. Ricerche bacteriologiche e chimiche sulle alterazioni del mais. Rivista Igiene Sanità Pubblica 1896; 7: 825-68

[93] Fleming A. The antibacterial action of cultures of a Penicillium, with special reference to their use in the isolation of $\mathrm{B}$. influenzae. Br J Experim Pathol 1929; 10: 226-36.

[94] Brown AG, Smale TC, King TJ, Hasenkamp R, Thompson RH. Crystal and molecular structure of compactin, a new antifungal metabolite from Penicillium brevicompactum. J Chem Soc Perkin Trans 1 1976; (11): 65-70.

[95] Endo A, Kuroda M, Tsujita Y. ML-236A, ML-236B, and ML236C, new inhibitors of cholesterogenesis produced by Penicillium citrinum. J Antibiot 1976; 29: 1346-8.
[96] Yu HS, Zhang L, Li L, et al. Recent developments and future prospects of antimicrobial metabolites produced by endophytes. Microbiol Res 2010; 165: 437-49.

[97] Kharwar RN, Mishra A, Gond SK, Stierle A, Stierle D. Anticancer compounds derived from fungal endophytes: their importance and future challenges. Nat Prod Rep 2011; 28: 1208-28

[98] Min YJ, Park MS, Fong JJ, Quan Y, Jung S, Lim YW. Diversity and saline resistance of endophytic fungi associated with Pinus thunbergii in coastal shelterbelts of Korea. J Microbiol Biotechnol 2014; 24(3): 324-33.

[99] Jena SK, Tayung K. Endophytic fungal communities associated with two ethno-medicinal plants of Similipal Biosphere Reserve, India and their antimicrobial prospective. J Appl Pharm Sci 2013; 3(4 Suppl 1): S7-12.

[100] Shiono Y, Seino Y, Koseki T, Murayama T, Kimura K. Antarones A and B, two polyketides from an endophytic Penicillium antarcticum. Z Naturforsch 2008; 63b: 909-14.

[101] Fill TP, Geris dos Santos RM, Barisson A, Rodrigues-Filho E, Souza AQL. Co-production of bisphenylpropanoid amides and meroterpenes by an endophytic Penicillium brasilianum found in the root bark of Melia azedarach. Z Naturforsch 2009; 64c: 355-60.

[102] Fisher PJ, Petrini O, Webster J. Aquatic hyphomycetes and other fungi in living aquatic and terrestrial roots of Alnus glutinosa. Mycol Res 1991; 95: 543-7.

[103] Vega FE, Simpkins A, Aime MC, et al. Fungal endophyte diversity in coffee plants from Colombia, Hawai'i, Mexico and Puerto Rico. Fungal Ecol 2010; 3: 122-38.

[104] Posada F, Aime MC, Peterson SW, Rehner SA, Vega FE. Inoculation of coffee plants with the fungal entomopathogen Beauveria bassiana (Ascomycota: Hypocreales). Mycol Res 2007; 111: 748-57

[105] Gazis R, Chaverri P. Diversity of fungal endophytes in leaves and stems of wild rubber trees (Hevea brasiliensis) in Peru. Fungal Ecol 2010; 3: 240-54.

[106] Unterseher M, Reiher A, Finstermeier K, Otto P, Morawetz W. Species richness and distribution patterns of leaf-inhabiting endophytic fungi in a temperate forest canopy. Mycol Progr 2007; 6: 201-12.

[107] Pelaez F, Collado J, Arenal F, et al. Endophytic fungi from plants living on gypsum soils as a source of secondary metabolites with antimicrobial activity. Mycol Res 1998; 102: 755-61.

[108] Kajula M, Tejesvi MV, Kolehmainen S, et al. The siderophore ferricrocin produced by specific foliar endophytic fungi in vitro. Fungal Biol 2010; 114: 248-54.

[109] Huang H, Feng X, Xiao Z, et al. Azaphilones and p-terphenyls from the mangrove endophytic fungus Penicillium chermesinum (ZH4-E2) isolated from the South China Sea. J Nat Prod 2011; 74: 997-1002.

[110] Gautam AK. Diversity of fungal endophytes in some medicinal plants of Himachal Pradesh, India. Arch Phytopathol Plant Prot 2014; 47: 537-44.

[111] Hormazabal E, Piontelli E. Endophytic fungi from Chilean native gymnosperms: antimicrobial activity against human and phytopathogenic fungi. World J Microbiol Biotechnol 2009; 25: 813-9.

[112] El-Morsy EM. Fungi isolated from the endorhizosphere of halophytic plants from the Red Sea Coast of Egypt. Fungal Diver 2000; 5: 43-54

[113] Anita DD, Sridhar KR. Assemblage and diversity of fungi associated with mangrove wild legume Canavalia cathartica. Tropic Subtropic Agroecosyst 2009; 10: 225-35.

[114] Mahmoud A-LE. Mycotoxin-producing potential of fungi associated with qat (Catha edulis) leaves in Yemen. Folia Microbiol 2000; 45: 452-6.

[115] Nicoletti R, De Filippis A, Buommino E. Antagonistic aptitude and antiproliferative properties on tumor cells of fungal endophytes from the Astroni Nature Reserve, Italy. Afr J Microbiol Res 2013; 7: 4073-83.

[116] Menkis A, Vasiliauskas R, Taylor AFS, Stenlid J, Finlay R. Fungal communities in mycorrhizal roots of conifer seedlings in forest nurseries under different cultivation systems, assessed by morphotyping, direct sequencing and mycelial isolation. Mycorrhiza 2005; 16: 33-41.

[117] Dhankhar S, Kumar S, Sandeep D, Yadav JP. Antioxidant activity of fungal endophytes isolated from Salvadora oleoides Decne. Int J Pharm Pharm Sci 2012; 4: 380-5. 
[118] Anita DD, Sridhar KR, Bhat R. Diversity of fungi associated with mangrove legume Sesbania bispinosa (Jacq.) W. Wight (Fabaceae). Livest Res Rural Dev 2009; 21: article \# 67. Available from: http://www.lrrd.org/lrrd21/5/anit21067.htm

[119] El-Nagerabi SAF, Elshafie AE, AlKhanjari SS. Endophytic fungi associated with Ziziphus species from mountainous area of Oman and new records. Biodiversitas 2013; 14: 10-6.

[120] Singh SB, Zinka DL, Guan Z, et al. Isolation, structure, and HIV-1 integrase inhibitory activity of xanthoviridicatin $\mathrm{E}$ and $\mathrm{F}$, two novel fungal metabolites produced by Penicillium chrysogenum. Helv Chim Acta 2003; 86: 3380-5.

[121] Lorenzi E, Lorando E, Picco AM. Microfunghi endofitici ed epifitici di Picea abies (L.) Karst. in ambiente naturale ed antropizzato in Lombardia. Forest@ 2006; 3: 426-36.

[122] Geris dos Santos RM, Rodrigues-Fo E, Caldas Rocha W, Teixeira MFS. Endophytic fungi from Melia azedarach. World J Microbiol Biotechnol 2003; 19: 767-70.

[123] Rosa LH, Gonçalves VN, Caligiorne RB, et al. Leishmanicidal, trypanocidal, and cytotoxic activities of endophytic fungi associated with bioactive plants in Brazil. Braz J Microbiol 2010; 41: 420-30.

[124] Gehlot P, Bohra NK, Purohit DK. Endophytic mycoflora of inner bark of Prosopis cineraria - a key stone tree species of Indian desert. Am-Euras J Bot 2008; 1; 1-4.

[125] Xiang Y, Lu A, Wu W. Identification of Taxus cuspidata Sieb. et Zucc. endophytic fungi-new species, species known and their metabolite. J For Res 2003; 14: 290-4.

[126] Miles LA, Lopera CA, González S, de García MC, Franco AE, Restrepo S. Exploring the biocontrol potential of fungal endophytes from an Andean Colombian Paramo ecosystem. BioControl 2012; 57: 697-710

[127] Yan HJ, Gao SS, Li CS, Li XM, Wang BG. Chemical constituents of a marine-derived endophytic fungus Penicillium commune G2M. Molecules 2010; 15: 3270-5.

[128] Hakizimana JD, Gryzenhout M, Coutinho TA, van den Berg N. Endophytic diversity in Persea americana (avocado) trees and their ability to display biocontrol activity against Phytophthora cinnamomi. Proc VII World Avocado Congr, Cairns, Australia, 5-9 Sept 2011. Avaliable from:

http://www.avocadosource.com/wac7/Section_01/HakizimanaJD20 11.pdf

[129] Frisvad JC, Samson RA. Polyphasic taxonomy of Penicillium subgenus Penicillium. A guide to identification of food and airborne terverticillate Penicillia and their mycotoxins. Stud Mycol 2004; 49: 1-173.

[130] Khan R, Shahzad S, Choudhary MI, Khan SA, Ahmad A. Communities of endophytic fungi in medicinal plant Withania somnifera. Pak J Bot 2010; 42: 1281-7.

[131] Verma VC, Gond SK, Kumar A, Kharwar RN, Boulanger LA, Strobel G. Endophytic fungal flora from roots and fruits of Indian neem plant Azadirachta indica, and impact of culture media on their isolation. Indian J Microbiol 2011; 51: 469-76.

[132] Kharwar RN, Gond SK, Kumar A, Mishra A. A comparative study of endophytic and epiphytic fungal association with leaf of Eucalyptus citriodora Hook., and their antimicrobial activity. World J Microbiol Biotechnol 2010; 26: 1941-8.

[133] Vega FE, Posada F, Aime MC, Peterson SW, Rehner SA. Fungal endophytes in green coffee seeds. Mycosystema 2008; 27: 75-84.

[134] Valente AM, Ferreira AG, Daolio C, et al. Production of 5-hydroxy-7-methoxy-4-methylphthalide in a culture of Penicillium crustosum. Anais Acad Bras Ciências 2013; 85: 48796.

[135] Wu H, Yang H, You X, Li Y. Isolation and characterization of saponin-producing fungal endophytes from Aralia elata in Northeast China. Int J Mol Sci 2012; 13: 16255-66.

[136] Bettucci L, Alonso R. A comparative study of fungal populations in healthy and symptomatic twigs of Eucalyptus grandis in Uruguay. Mycol Res 1997; 101: 1060-4.

[137] Kayini A, Pandey RR. Phyllosphere fungi of Alnus nepalensis, Castanopsis hystrix and Schima walichii in a subtropical forest of North East India. J Am Sci 2010; 6: 118-24.

[138] Lu Z, Zhu H, Fu P, et al. Cytotoxic polyphenols from the marinederived fungus Penicillium expansum. J Nat Prod 2010; 73: 911-4.

[139] Kim CS, Park MS, Yu SH. Two species of endophytic Penicillium from Pinus rigida in Korea. Mycobiology 2008; 36: 222-7.
[140] Hoffman MT, Arnold AE. Geographic locality and host identity shape fungal endophyte communities in cupressaceous trees. Mycol Res 2008; 112: 331-44.

[141] Vujanovic V, Brisson J. A comparative study of endophytic mycobiota in leaves of Acer saccharum in eastern North America. Mycol Progr 2002; 1: 147-54.

[142] Cabezas L, Calderon C, Medina LM, et al. Characterization of cellulases of fungal endophytes isolated from Espeletia spp. J Microbiol 2012; 50: 1009-13.

[143] Fisher PJ, Petrini O, Sutton BC. A comparative study of fungal endophytes in leaves, xylem and bark of Eucalyptus nitens in Australia and England. Sydowia 1993; 45: 338-45.

[144] Lodge DJ, Fisher PJ, Sutton BC. Endophytic fungi of Manilkara bidentata leaves in Puerto Rico. Mycologia 1996; 88: 733-8.

[145] Collado J, Platas G, Gonzalez I, Pelaez F. Geographical and seasonal influences on the distribution of fungal endophytes in Quercus ilex. New Phytol 1999; 144: 525-32.

[146] Fisher PJ, Petrini O, Petrini LE, Sutton BC. Fungal endophytes from the leaves and twigs of Quercus ilex L. from England, Majorca and Switzerland. New Phytol 1994; 127: 133-7.

[147] Evans HC, Holmes KA, Thomas SE. Endophytes and mycoparasites associated with an indigenous forest tree, Theobroma gileri, in Ecuador and a preliminary assessment of their potential as biocontrol agents of cocoa diseases. Mycol Progr 2003; 2: 149-60.

[148] Sieber-Canavesi F, Sieber TN. Successional patterns of fungal communities in needles of European silver fir (Abies alba Mill.). New Phytol 1993; 125: 149-61.

[149] Sieber T, Hugentobler C. Endophytische pilze in blättern und ästen gesunder und geschädigter buchen (Fagus sylvatica L.). Eur J For Pathol 1987; 17: 411-25.

[150] Müller MM, Hallaksela AM. Diversity of Norway spruce needle endophytes in various mixed and pure Norway spruce stands. Mycol Res 1998; 102: 1183-9.

[151] Koukol O, Kolařík M, Kolářová Z, Baldrian P. Diversity of foliar endophytes in wind-fallen Picea abies trees. Fungal Diver 2012; 54: 69-77.

[152] Stefani FOP, Bérubé JA. Biodiversity of foliar fungal endophytes in white spruce (Picea glauca) from southern Québec. Can J Bot 2006; 84: 777-90.

[153] Houbraken J, Frisvad JC, Seifert KA, et al. New penicillinproducing Penicillium species and an overview of section Chrysogena. Persoonia 2012; 29: 78-100.

[154] Schmeda-Hirschmann G, Hormazabal E, Astudillo L, Rodriguez J, Theoduloz C. Secondary metabolites from endophytic fungi isolated from the Chilean gymnosperm Prumnopitys andina (Lleuque). World J Microbiol Biotechnol 2005; 21: 27-32.

[155] Tayung K, Jha DK. Antimicrobial endophytic fungal assemblages inhabiting bark of Taxus baccata L. of Indo-Burma mega biodiversity hotspot. Indian J Microbiol 2010; 50: S74-81.

[156] Murali TS, Suryanarayanan TS, Venkatesan G. Fungal endophyte communities in two tropical forests of southern India: diversity and host affiliation. Mycol Progr 2007; 6: 191-9.

[157] Bergero R, Perotto S, Girlanda M, Vidano G, Luppi AM. Ericoid mycorrhizal fungi are common root associates of a Mediterranean ectomycorrhizal plant (Quercus ilex). Mol Ecol 2000; 9: 1639-49.

[158] Wu L, Han T, Li W, et al. Geographic and tissue influences on endophytic fungal communities of Taxus chinensis var. mairei in China. Curr Microbiol 2013; 66(1): 40-8.

[159] Faure-Raynaud M, Daniere C, Moiroud A, Capellano A. Intraspecific variability of isozymes in Penicillium nodositatum Valla, a fungus inducing 'myconodules' on the root-system of Alnus sp. Plant Soil 1991; 137: 285-91.

[160] Lucero ME, Barrowa JR, Osunab P, Reyes I. Plant-fungal interactions in arid and semi-arid ecosystems: Large-scale impacts from microscale processes. J Arid Environ 2006; 65: 276-84.

[161] Posada F, Vega FE. Establishment of the fungal entomopathogen Beauveria bassiana (Ascomycota: Hypocreales) as an endophyte in cocoa seedlings (Theobroma cacao). Mycologia 2005; 97: 1195200.

[162] Chandrasekar S, Thiyagarajan S, Sridhar R, Ambethkar B. Diversity of endophytic mycobiota colonizing the aerial tissues of Thuja plicata (Donn ex. D. Don.). Int J Curr Microbiol Appl Sci 2013; 2: 176-83.

[163] Phongpaichit S, Rungjindamai N, Rukachaisirikul V, Sakayaroj J. Antimicrobial activity in cultures of endophytic fungi isolated from 
Garcinia species. FEMS Immunol Med Microbiol 2006; 48: 36772.

[164] Ding GZ, Liu J, Wang JM, Fang L, Yu SS. Secondary metabolites from the endophytic fungi Penicillium polonicum and Aspergillus fumigatus. J Asian Nat Prod Res 2013; 15: 446-52.

[165] Stierle A, Strobel G, Stierle D, Grothaus P, Bignami G. The search for a taxol-producing microorganism among the endophytic fungi of the Pacific yew, Taxus brevifolia. J Nat Prod 1995; 58: 1315-24.

[166] Vaz ABM, Brandão LR, Vieira MLA, et al. Diversity and antimicrobial activity of fungal endophyte communities associated with plants of Brazilian savanna ecosystems. Afr J Microbiol Res 2012; 6: 3173-85.

[167] Fang W, Yang L, Zhu X, Zeng L, Li X. Seasonal and habitat dependent variations in culturable endophytes of Camellia sinensis. J Plant Pathol Microbiol 2013; 4(3): 169; doi:10.4172/21577471.1000169

[168] Phongpaichit S, Nikom J, Rungjindamai N, et al. Biological activities of extracts from endophytic fungi isolated from Garcinia plants. FEMS Immunol Med Microbiol 2007; 51: 517-25.

[169] Khan R, Shahzad S, Choudhary MI, Khan SA, Ahmad A. Biodiversity of the endophytic fungi isolated from Calotropis procera (Ait.) R. Br. Pak J Bot 2007; 39: 2233-9.

[170] Houbraken J, Frisvad JC, Samson RA. Taxonomy of Penicillium section Citrina. Stud Mycol 2011; 70; 53-138.

[171] Chen G, Zhu Y, Wang HZ, Wang SJ, Zhang RQ. The metabolites of a mangrove endophytic fungus, Penicillium thomi. J Asian Nat Prod Res 2007; 9(2): 159-64.

[172] Shoeb M, Thoo-Lin PK, Nahar N. Anti-colon cancer activity of endophytic fungal strains from Terminalia chebula Rezt. Bangladesh J Pharmacol 2012; 7(1): 47-9.

[173] Gennaro M, Gonthier P, Nicolotti G. Fungal endophytic communities in healthy and declining Quercus robur L. and $Q$. cerris L. trees in northern Italy. J Phytopathol 2003; 151: 529-34.

[174] Syed S, Riyaz-Ul-Hassan S, Johri S. A novel cellulase from an endophyte, Penicillium sp. NFCCI 2862. Am J Microbiol Res 2013; 1(4): 84-91.

[175] Nagaraja TG, Devkar PG. Seasonal occurrence of endophytic mycoflora of inner bark of medicinal plant Acacia catechu Willd. Bioscan 2010; 5: 243-5.

[176] Maria GL, Sridhar KR. Endophytic fungal assemblage of two halophytes from west coast mangrove habitats, India. Czech Mycol 2003; 55: 241-51.

[177] Yan J, Cui Y, Ding J, Zhou L, Cheng Z, Zhang M. Isolation and identification of the causal pathogens for kiwifruit bacterial canker and the isolation of the antagonistic endophytic fungi from kiwifruit in Sichuan, China. J Agr Sci 2013; 5: 262-8.

[178] Raviraja NS. Fungal endophytes in five medicinal plant species from Kudremukh Range, Western Ghats of India. J Basic Microbiol 2005; 45: 230-5.

[179] Xu M, Gessner G, Groth I, et al. Shearinines D-K, new indole triterpenoids from an endophytic Penicillium sp. (strain HKI0459) with blocking activity on large-conductance calcium-activated potassium channels. Tetrahedron 2007; 63: 435-44.

[180] Lin ZJ, Lu ZY, Zhu TJ, Fang YC, Gu QQ, Zhu WM. Penicillenols from Penicillium sp. GQ-7, an endophytic fungus associated with Aegiceras corniculatum. Chem Pharm Bull 2008; 56: 217-21.

[181] Lin Z, Zhu T, Fang Y, Gu Q, Zhu W. Polyketides from Penicillium sp. JP-1, an endophytic fungus associated with the mangrove plant Aegiceras corniculatum. Phytochemistry 2008; 69: 1273-8.

[182] Cai X, Liang X, Zhou S, et al. Cytotoxic and topoisomerase I inhibitory activities from extracts of endophytic fungi isolated from mangrove plants in Zhuhai, China. J Ecol Nat Environ 2010; 2: 1724.

[183] Gond SK, Verma VC, Kumar A, Kumar V, Kharwar RN. Study of endophytic fungal community from different parts of Aegle marmelos Correae (Rutaceae) from Varanasi (India). World J Microbiol Biotechnol 2007; 23: 1371-5.

[184] Chareprasert S, Piapukiew J, Thienhirun S, Whalley AJS, Sihanonth P. Endophytic fungi of teak leaves Tectona grandis L. and rain tree leaves Samanea saman Merr. World J Microbiol Biotechnol 2006; 22: 481-6.

[185] Mahapatra S, Banerjee D. Diversity and screening for antimicrobial activity of endophytic fungi from Alstonia scholaris. Acta Microbiol Immunol Hung 2010; 57: 215-23.

[186] de Oliveira Silva RL, Luz JS, da Silveira EB, Cavalcante UMT. Fungos endofíticos em Annona spp.: isolamento, caracterização enzimática e promoção do crescimento em mudas de pinha (Annona squamosa L.). Acta Bot Bras 2006; 20: 649-55.

[187] Hsieh PW, Hsu LC, Lai CH, et al. Evaluation of the bioactivities of extracts of endophytes isolated from Taiwanese herbal plants. World J Microbiol Biotechnol 2009; 25: 1461-9.

[188] Jeewon R, Ittoo J, Mahadeb D, Jaufeerally-Fakim Y, Wang HK, Liu AR. DNA based identification and phylogenetic characterisation of endophytic and saprobic fungi from Antidesma madagascariense, a medicinal plant in Mauritius. J Mycol 2013; doi: org/10.1155/2013/781914

[189] Gong LJ, Guo SX. Endophytic fungi from Dracaena cambodiana and Aquilaria sinensis and their antimicrobial activity. Afr $\mathbf{J}$ Biotechnol 2009; 8: 731-6.

[190] Kumaresan V, Suryanarayanan TS. Occurrence and distribution of endophytic fungi in a mangrove community. Mycol Res 2001; 105: 1388-91.

[191] Mahesh B, Tejesvi MV, Nalini MS, et al. Endophytic mycoflora of inner bark of Azadirachta indica A. Juss. Curr Sci 2005; 88: 218-9.

[192] Verma VC, Gond SK, Kumar A, Kharwar RN, Strobel G. The endophytic mycoflora of bark, leaf, and stem tissues of Azadirachta indica A. Juss (Neem) from Varanasi (India). Microb Ecol 2007; 54: 119-25.

[193] Tenguria RK, Khan FN. Distribution of endophytic fungi in leaves of Azadirachta indica A. Juss. (Neem) of Panchmarhi biosphere reserve. Curr Bot 2011; 2(2): 27-9.

[194] Bagchi B, Banerjee D. Diversity of fungal endophytes in Bauhinia vahlii (a lianas) from different regions of Paschim Medinipur district of West Bengal. Int J Sci Environ Technol 2013; 2: 748-56.

[195] Han Z, Mei W, Zhao Y, Deng Y, Dai H. A new cytotoxic isocoumarin from endophytic fungus Penicillium sp. 091402 of the mangrove plant Bruguiera sexangula. Chem Nat Comp 2009; 45: 805-7.

[196] Sunitha VH, Devi DN, Srinivas C. Extracellular enzymatic activity of endophytic fungal strains isolated from medicinal plants. World J Agr Sci 2013; 9: 1-9.

[197] Bhagobaty RK, Joshi SR. Multi-loci molecular characterisation of endophytic fungi isolated from five medicinal plants of Meghalaya, India. Mycobiology 2011;39(2): 71-8.

[198] Zhou Z, Zhang C, Zhou W, et al. Diversity and plant growthpromoting ability of endophytic fungi from the five flower plant species collected from Yunnan, Southwest China. J Plant Interact 2014; 9: 585-91.

[199] Agusta A, Ohashi K, Shibuya H. Composition of the endophytic filamentous fungi isolated from the tea plant Camellia sinensis. J Nat Med 2006; 60: 268-72.

[200] Ding T, Jiang T, Zhou J, Xu L, Gao ZM. Evaluation of antimicrobial activity of endophytic fungi from Camptotheca acuminata (Nyssaceae). Gen Mol Res 2010; 9: 2104-12.

[201] Bayman P, Angulo-Sandoval P, Báez-Ortiz Z, Lodge DJ. Distribution and dispersal of Xylaria endophytes in two tree species in Puerto Rico. Mycol Res 1998; 102: 944-8.

[202] Han Z, Mei WL, Cui HB, et al. Antibacterial constituents from the endophytic fungus Penicillium sp. of mangrove plant Cerbera manghas. Chem J Chin Univer 2008; 29: 749-52.

[203] Jin PF, Dai HF, Zuo WJ, Zeng YB, Guo ZK, Mei WL. Metabolites from the endophytic fungus of Ceriops tagal. Chin J Med Chem 2013; 23: 309-11.

[204] Wang T, Wei S, Wei Q, Hou M, Cui X. Diversity of endophytic fungi from leaves of Cinnamomum longepaniculatum N. Chao ex H.W. Li in Yibin, Sichuan, China. J Yunnan Univ (Nat Sci Ed) 2007; 3: 17.

[205] Santamaría J, Bayman P. Fungal epiphytes and endophytes of coffee leaves (Coffea arabica). Microb Ecol 2005; 50: 1-8.

[206] Gamboa-Gaitán MA. Presence of Aspergillus and other fungal symbionts in coffee beans from Colombia. Acta Biol Colomb 2012; 17: 39-50.

[207] Mulaw TB, Druzhinina IS, Kubicek CP, Atanasova L. Novel endophytic Trichoderma spp. isolated from healthy Coffea arabica roots are capable of controlling coffee tracheomycosis. Diversity 2013; 5: 750-66.

[208] Toofanee SB, Dulymamode R. Fungal endophytes associated with Cordemoya integrifolia. Fungal Diver 2002; 11: 169-75.

[209] Sutjaritvorakul T, Whalley AJS, Sihanonth P, Roengsumran S. Antimicrobial activity from endophytic fungi isolated from plant leaves in Dipterocarpous forest at Viengsa district Nan province, Thailand. J Agr Technol 2010; 6: 309-15. 
[210] Li JT, Fu XL, Tan C, Zeng Y, Wang Q, Zhao PJ. Two new chroman derivations from the endophytic Penicillium sp. DCS523. Molecules 2011; 16: 686-93.

[211] Douanla-Meli C, Langer E. Diversity and molecular phylogeny of fungal endophytes associated with Diospyros crassiflora. Mycology: Int J Fungal Biol 2012; 3: 175-87.

[212] Sbravatti Júnior JA, Auer CG, Pimentel IC, Santos ÁD, Schultz B. In vitro selection of endophytes for biological control of Botrytis cinerea in Eucalyptus benthamii. Floresta 2013; 43(1): 145-51.

[213] Bettucci L, Saravay M. Endophytic fungi of Eucalyptus globulus: a preliminary study. Mycol Res 1993; 97: 679-82.

[214] Sánchez Márquez S, Bills GF, Zabalgogeazcoa I. Fungal species diversity in juvenile and adult leaves of Eucalyptus globulus from plantations affected by Mycosphaerella leaf disease. Ann Appl Biol 2011; 158: 177-87.

[215] Unterseher M, Schnittler M. Dilution-to-extinction cultivation of leaf-inhabiting endophytic fungi in beech (Fagus sylvatica L.) Different cultivation techniques influence fungal biodiversity assessment. Mycol Res 2009; 113: 645-54.

[216] Zheng JH, Kang JC, Lei BX, Li QR, Wen TC, Meng ZB. Diversity of endophytic fungi associated with Ginkgo biloba. Mycosystema 2013; 32: 671-81.

[217] Yuan Y, Tian JM, Xiao J, Shao Q, Gao JM. Bioactive metabolites isolated from Penicillium sp. YY-20, the endophytic fungus from Ginkgo biloba. Nat Prod Res 2014; 28: 278-81.

[218] Davis RA, Andjic V, Kotiw M, Shivas RG. Phomoxins B and C: polyketides from an endophytic fungus of the genus Eupenicillium. Phytochemistry 2005; 66: 2771-5.

[219] Yuan LC, Zhao Q, Zhang KH, et al. Isolation and identification of endophytes from Jatropha curcas by ITS analysis. Southwest China J Agr Sci 2009; 22: 95-8.

[220] Guo Z, Cheng F, Zou K, Wang J, She Z, Lin Y. Secondary metabolites from the mangrove endophytic fungus Penicillium sp. (SBE-8). Nat Prod Commun 2009; 4: 1481-3.

[221] Huang X, Sun X, Ding B, et al. A new anti-acetylcholinesterase $\alpha$-pyrone meroterpene, arigsugacin I, from mangrove endophytic fungus Penicillium sp. sk5GW1L of Kandelia candel. Planta Med 2013; 79: 1572-5.

[222] Maheswari S, Rajagopal K. Biodiversity of endophytic fungi in Kigelia pinnata during two different seasons. Curr Sci 2013; 104 : 515-8.

[223] Johnston PR. Leaf endophytes of manuka (Leptospermum scoparium). Mycol Res 1998; 102: 1009-16.

[224] Steinke E, Williams PG, Ashford AE. The structure and fungal associates of mycorrhizas in Leucopogon parviflorus (Andr.) Lindl. Ann Bot 1996; 77: 413-9.

[225] Hata K, Atari R, Sone K. Isolation of endophytic fungi from leaves of Pasania edulis and their within-leaf distributions. Mycoscience 2002; 43: 369-73.

[226] Verma SK, Gond SK, Mishra A, et al. Impact of environmental variables on the isolation, diversity and antibacterial activity of endophytic fungal communities from Madhuca indica Gmel. at different locations in India. Ann Microbiol 2014; 64: 721-34.

[227] Koolen Ferreira HH, Ribeiro Soares E, Moura Araújo da Silva F, et al. An antimicrobial alkaloid and other metabolites produced by Penicillium sp. an endophytic fungus isolated from Mauritia flexuosa L. f. Quim Nova 2012; 35: 771-4.

[228] Pharamat T, Palaga T, Piapukiew J, Whalley AJS, Sihanonth P. Antimicrobial and anticancer activities of endophytic fungi from Mitrajyna javanica Koord and Val. Afr J Microbiol Res 2013; 7: 5565-72.

[229] Dhanalakshmi R, Umamaheswari S, Sugandhi P, Prasanth DA. Biodiversity of the endophytic fungi isolated from Moringa oleifera of Yercaud hills. Int J Pharm Sci Res 2013; 4: 1064-8.

[230] Zheng LP, Zhang Z, Xie LQ, Yuan HY, Zhang YQ. Antifungal activity of endophyte cultures of Morus alba L. against phytopathogenic fungi. Advan Mater Res 2013; 641-642: 615.

[231] Musavi SF, Balakrishnan RM. Biodiversity, antimicrobial potential, and phylogenetic placement of an endophytic Fusarium oxysporum NFX 06 isolated from Nothapodytes foetida. J Mycol 2013; doi: 10.1155/2013/172056

[232] Gond SK, Mishra A, Sharma VK, et al. Diversity and antimicrobial activity of endophytic fungi isolated from Nyctanthes arbor-tristis, a well-known medicinal plant of India. Mycoscience 2012; 53: 113-21.
[233] de Abreu LM, Almeida AR, Salgado M, Pfenning LH. Fungal endophytes associated with the mistletoe Phoradendron perrottettii and its host tree Tapirira guianensis. Mycol Progr 2010; 9: 559-66.

[234] Hoff JA, Klopfenstein NB, McDonald GI, et al. Fungal endophytes in woody roots of Douglas-fir (Pseudotsuga menziesii) and ponderosa pine (Pinus ponderosa). For Pathol 2004; 34: 255-71.

[235] Zamora P, Martínez-Ruiz C, Diez JJ. Fungi in needles and twigs of pine plantations from northern Spain. Fungal Diver 2008; 30: 17184.

[236] Helander ML. Responses of pine needle endophytes to air pollution. New Phytol 1995; 131: 223-9.

[237] Giordano L, Gonthier P, Varese GC, Miserere L, Nicolotti G. Mycobiota inhabiting sapwood of healthy and declining Scots pine (Pinus sylvestris L.) trees in the Alps. Fungal Diver 2009; 38: 6983.

[238] Suryanarayanan TS, Thennarasan S. Temporal variation in endophyte assemblages of Plumeria rubra leaves. Fungal Diver 2004; 15: 197-204.

[239] Busby PE, Zimmerman N, Weston DJ, Jawdy SS, Houbraken J, Newcombe G. Leaf endophytes and Populus genotype affect severity of damage from the necrotrophic leaf pathogen, Drepanopeziza populi. Ecosphere 2013; 4(10): article \# 125.

[240] Unterseher M, Petzold A, Schnittler M. Xerotolerant foliar endophytic fungi of Populus euphratica from the Tarim River basin, Central China are conspecific to endophytic ITS phylotypes of Populus tremula from temperate Europe. Fungal Diver 2012; 54 : 133-142.

[241] Santamaría O, Diez JJ. Fungi in leaves, twigs and stem bark of Populus tremula from northern Spain. For Pathol 2005; 35: 95-104.

[242] Albrectsen BR, Björkén L, Varad A, et al. Endophytic fungi in European aspen (Populus tremula) leaves-diversity, detection, and a suggested correlation with herbivory resistance. Fungal Diver 2010; 41: 17-28.

[243] Boncaldo E, Sicoli G, Mannerucci F, Luisi N. Caratterizzazione delle comunità fungine endofitiche di querce caducifoglie in Italia meridionale. Italia For Mont 2008; 63: 321-32.

[244] Anselmi N, Mazzaglia A. Correlation between the incidence of endophytic pathogenic fungi and oak decline in Quercus ilex L. after fire damages. IOBC/WPRS Bull 2005; 28(8): 93-9.

[245] Li HY, Shen M, Zhou ZP, et al. Diversity and cold adaptation of endophytic fungi from five dominant plant species collected from the Baima Snow Mountain, Southwest China. Fungal Diver 2012; 54: 79-86.

[246] Kaus A, Schmitt V, Simon A, Wild A. Microscopial and mycological investigations on wood of peduncolate oak (Quercus robur L.) relative to the occurrence of oak decline. J Plant Physiol 1996; 148: 302-8.

[247] Franceschini A, Maddau L, Marras F. Incidence d'endophytes fongiques impliqués dans le dépérissement du chêne-liège. IOBC/WPRS Bull 2002; 25(5): 29-36.

[248] Wang FW, Jiao RH, Cheng AB, Tan SH, Song YC. Antimicrobial potentials of endophytic fungi residing in Quercus variabilis and brefeldin A obtained from Cladosporium sp. World J Microbiol Biotechnol 2007; 23: 79-83.

[249] Nath A, Chattopadhyay A, Joshi SR. Biological activity of endophytic fungi of Rauwolfia serpentina Benth: an ethnomedicinal plant used in folk medicines in Northeast India. Proc Nat Acad Sci, India Sect B: Biol Sci 2013; 83; doi: 10.1007/s40011-013-0184-8

[250] Sathiya Rathna G, Elavarasi A, Peninal S, Subramanian J, Mano G, Kalaiselvam M. Extracellular biosynthesis of silver nanoparticles by endophytic fungus Aspergillus terreus and its antidermatophytic activity. Int J Pharm Biol Arch 2013; 4: 481-7.

[251] Suryanarayanan TS, Kumaresan V, Johnson JA. Foliar fungal endophytes from two species of the mangrove Rhizophora. Can J Microbiol 1998; 44: 1003-6.

[252] Prihanto AA, Firdaus M, Nurdiani R. Endophytic fungi isolated from mangrove (Rhyzopora mucronata) and its antibacterial activity on Staphylococcus aureus and Escherichia coli. J Food Sci Engin 2011; 1: 386-9.

[253] Baral B, Rana P, Maharjan BL. Antimicrobial potentials of endophytic fungi inhabiting Rhododendron anthopogon D. Don. Ecoprint 2011; 18: 39-44.

[254] Sun SS, Chen XM, Guo SX. Analysis of endophytic fungi in roots of Santalum album Linn and its host plant Kuhnia rosmarinifolia 
Vent. J Zhejiang Univ-Sci B (Biomed Biotechnol) 2014; 15: 10915.

[255] Ohashi K, Miyagawa Y, Nakamura Y, Shibuya H. Bioproduction of bile acids and the glycine conjugates by Penicillium fungus. $\mathrm{J}$ Nat Med 2008; 62: 83-6.

[256] Govindappa M, Channabasava R, Sunil Kumar KR, Pushpalatha KC. Antioxidant activity and phytochemical screening of crude endophytes extracts of Tabebuia argentea Bur. \& K. Sch. Am J Plant Sci 2013; 4: 1641-52.

[257] Caruso M, Colombo AL, Fedeli L, et al. Isolation of endophytic fungi and actinomycetes taxane producers. Ann Microbiol 2000; 50: 3-13.

[258] Kawamura H, Kaneko T, Koshino H, Esumi Y, Uzawa J, Sugawara F. Penicillides from Penicillium sp. isolated from Taxus cuspidata. Nat Prod Lett 2000; 14: 477-84.

[259] Rivera-Orduña FN, Suarez-Sanchez RA, Flores-Bustamante ZR, Gracida-Rodriguez JN, Flores-Cotera LB. Diversity of endophytic fungi of Taxus globosa (Mexican yew). Fungal Diver 2011; 47: 6574.

[260] Tejesvi MV, Mahesh B, Nalini MS, et al. Endophytic fungal assemblages from inner bark and twig of Terminalia arjuna W. \& A. (Combretaceae). World J Microbiol Biotechnol 2005; 21: 153540.

[261] Sadananda TS, Govindappa M, Ramachandra YL. Isolation and characterization of D-galactose, $\mathrm{N}$-acetylgalactosamine, fructose, maltose specific lectin from eight different endophytic fungi of Viscum album L. Asian J Biomed Pharm Sci 2013; 3(26): 11-20.

[262] Casella TM, Eparvier V, Mandavid H, et al. Antimicrobial and cytotoxic secondary metabolites from tropical leaf endophytes: Isolation of antibacterial agent pyrrocidine $\mathrm{C}$ from Lewia infectoria SNB-GTC2402. Phytochemistry 2013; 96: 370-7.

[263] Kirchmair M, Trenkwalder S, Huber L, Neuhauser S. Endophytes from healthy and esca diseased vines. Phytopathol Medit 2010; 49: 119.

[264] González V, Tello ML. The endophytic mycota associated with Vitis vinifera in central Spain. Fungal Diver 2011; 47: 29-42.

[265] Núñez-Trujillo G, Cabrera R, Burgos-Reyes RL, et al. Endophytic fungi from Vitis vinifera $\mathrm{L}$. isolated in Canary Islands and Azores as potential biocontrol agents of Botrytis cinerea Pers.: Fr. J Hortic For Biotechnol 2012; 16(1): 1-6.

[266] Fill Pacheco T, Pereira GK, Geris dos Santos RM, Rodrigues-Fo E. Four additional meroterpenes produced by Penicillium sp. found in association with Melia azedarach. Possible biosynthetic intermediates to austin. Z Naturforsch 2007; 62b: 1035-44.
[267] Geris dos Santos RM, Rodrigues-Fo E. Further meroterpenes produced by Penicillium sp., an endophyte obtained from Melia azedarach. Z Naturforsch 2003; 58c: 663-9.

[268] Geris dos Santos RM, Rodrigues-Fo E. Structures of meroterpenes produced by Penicillium sp., an endophytic fungus found associated with Melia azedarach. J Braz Chem Soc 2003; 14: 7227.

[269] Yang J, Huang R, Qiu SX, She Z, Lin Y. A new isobenzofuranone from the mangrove endophytic fungus Penicillium sp. (ZH58). Nat Prod Res 2013; 27: 1902-5.

[270] Marinho AMR, Marinho PSB, Santos LS, Rodrigues Filho E, Ferreira ICP. Active polyketides isolated from Penicillium herquei. Anais Acad Bras Ciências 2013; 85: 909-12.

[271] Marinho AMR, Rodrigues-Filho E, Moitinho MLR, Santos LS. Biologically active polyketides produced by Penicillium janthinellum isolated as an endophytic fungus from fruits of Melia azedarach. J Braz Chem Soc 2005; 16: 280-3.

[272] Pastre R, Marinho AMR, Rodrigues-Filho E, Souza AQL, Odair Pereira J. Diversidade de policetídeos produzidos por espécies de Penicillium isoladas de Melia azedarach e Murraya paniculata. Quim Nova 2007; 30: 1867-71.

[273] Arunpanichlert J, Rukachaisirikul V, Sukpondma Y, et al. Azaphilone and isocoumarin derivatives from the endophytic fungus Penicillium sclerotiorum PSU-A13. Chem Pharm Bull 2010; 58: 1033-6.

[274] Marinho AMR, Rodrigues-Filho E. Dicitrinol, a citrinin dimer, produced by Penicillium janthinellum. Helv Chim Acta 2011; 94: 835-41.

[275] da Silva JS, Fill Pacheco T, Ferreira da Silva B, Rodrigues-Fo E. Diclavatol and tetronic acids from Penicillium griseoroseum. Nat Prod Res 2013; 27: 9-16.

[276] Marinho AMR, Rodrigues-Filho E, Ferreira AG, Santos LS. C25 steroid epimers produced by Penicillium janthinellum, a fungus isolated from fruits of Melia azedarach. J Braz Chem Soc 2005; 16: $1342-6$.

[277] Rukachaisirikul V, Kaeobamrung J, Panwiriyarat W, et al. A new pyrone derivative from the endophytic fungus Penicillium paxilli PSU-A71. Chem Pharm Bull 2007; 55: 1383-4.

[278] Davis RA, Longden J, Avery VM, Healy PC. The isolation, structure determination and cytotoxicity of the new fungal metabolite, trichodermamide C. Bioorg Med Chem Lett 2008; 18 2836-9.

[279] Fill Pacheco T, Briganti Rodrigues Asenha H, Marques AS, Ferreira AG, Rodrigues-Fo E. Time course production of indole alkaloids by an endophytic strain of Penicillium brasilianum cultivated in rice. Nat Prod Res 2013; 27: 967-74.

(C) Nicoletti et al.; Licensee Bentham Open.

This is an open access article licensed under the terms of the Creative Commons Attribution Non-Commercial License (http://creativecommons.org/ licenses/by-nc/3.0/), which permits unrestricted, non-commercial use, distribution and reproduction in any medium, provided the work is properly cited. 